\title{
DELTA-STRUCTURES ON MAPPING CLASS GROUPS AND BRAID GROUPS
}

\author{
A. J. BERRICK, E. HANBURY, AND J. WU
}

\begin{abstract}
We describe a Delta-group structure on the mapping class groups of surfaces, and show that it is compatible with the Delta-group structures of the braid groups of surfaces given by Berrick-Cohen-Wong-Wu. We then prove an isomorphism theorem relating these two Delta-groups. This is the first of a pair of papers on this topic.
\end{abstract}

\section{INTRODUCTION AND STATEMENT OF RESUlTS}

The combinatorial structure of braid groups has revealed a wealth of information in the past decade. In [20], the third author was able to describe $\pi_{n}\left(S^{2}\right)$ as the fixed point set of a particular action of the pure Artin braid group $P_{n}\left(D^{2}\right)$. Then in [1], $\pi_{n}\left(S^{2}\right)$ was shown to appear in an exact sequence as the cokernel of a map on braid groups induced by the inclusion of the disc $D^{2}$ in the 2 -sphere. That work also showed that a particular combinatorial apparatus, known as a $\Delta$-group, exists for the braid groups of any surface $M$. For a survey, including work of [21, 14] and [5], see [6].

Now, it is well known that there is a close relation between braid groups and mapping class groups of surfaces. Indeed, in the case of the disc, the pure braid group is isomorphic to the corresponding mapping class group: $P_{n}\left(D^{2}\right) \cong \Gamma^{n}\left(D^{2}\right)$ for all $n \geq 1$. It is therefore natural to seek to define a $\Delta$-group structure for the mapping class groups of an arbitrary surface $M$, and to explore its relation to the $\Delta$-group structure on the groups $P_{n}(M)$. That is the purpose of the present work.

A companion paper [3] uses the results from this paper to calculate the homotopy groups of these $\Delta$-groups and to generalize the exact sequence of [1] to arbitrary surfaces and study its counterpart in the setting of mapping class groups.

To present our main results, we need some notation. We work with a general surface $M$, possibly with boundary, and having a number of marked points (punctures). The combinatorial structure arises by varying the number of marked points. First, for $k \geq 1$, recall the configuration space

$$
\operatorname{Conf}_{k}(M)=\left\{\left(x_{0}, \ldots, x_{k-1}\right) \in M^{k} \mid x_{i} \neq x_{j} \text { if } i \neq j\right\}
$$

Received by the editors January 20, 2012 and, in revised form, May 1, 2012 and May 29, 2012. 2010 Mathematics Subject Classification. Primary 20F36; Secondary 55Q40, 55R80, 55U10, 57M07, 57S05.

The authors gratefully acknowledge the assistance of NUS research grants R-146-000-097-112, R-146-000-101-112 and R-146-000-137-112. 
of $k$-tuples of distinct points on $M$. Its fundamental group is the $k$ th pure braid group of $M$ :

$$
P_{k}(M)=\pi_{1}\left(\operatorname{Conf}_{k}(M)\right) .
$$

In [1] the authors show that the sequence $\underline{P}(M)=\left\{P_{1}(M), P_{2}(M), \ldots\right\}$ is a $\Delta$ group for all $M$; that is, for each $i=0, \ldots, k$ there is a homomorphism $d_{i}$ : $P_{k+1}(M) \rightarrow P_{k}(M)$, and these maps satisfy the face map identity $d_{j} d_{i}=d_{i} d_{j+1}$ when $i \leq j$. Topologically, the map $d_{i}$ corresponds to removing the $i$ th component of a loop in the configuration space $\operatorname{Conf}_{k}(M) \subseteq M^{k}$. Geometrically, this operation can be described as removing the $i$ th strand of a braid (and then renumbering).

$\Delta$-groups can be considered as combinatorial models of spaces; any $\Delta$-group $\underline{G}$ has an associated sequence of homotopy sets $\pi_{*}(\underline{G})$. The paper [1] shows that the homotopy sets of $\underline{P}\left(S^{2}\right)$ are in fact groups, and are isomorphic to the homotopy groups $\pi_{k}\left(S^{2}\right)$ for $k \geq 4$. This gives a new description of the homotopy groups of $S^{2}$ in terms of braids; it has the advantage that the homotopy groups of $\underline{P}\left(S^{2}\right)$ can be defined combinatorially. Information about stable homotopy groups of spheres also arises in this way: it is known that $\pi_{i}\left(S^{n}\right)$ and $\pi_{i-n+3}\left(S^{3}\right)$ have isomorphic $p$ primary components for $n \geq 3$ odd, $p$ prime and $i<4 p+n-6$ (see e.g. 18, Corollary 9.7.12]). In particular, for odd $p$, the elements of order $p$ in the stable $(2 p-3)$-stem are suspensions of elements of order $p$ in $\pi_{2 p}\left(S^{3}\right)$. Recall that $\pi_{k}\left(S^{3}\right) \cong \pi_{k}\left(S^{2}\right)$ for $k \geq 3$ from the classical Hopf fibration.

On the other hand, the $k$ th pure mapping class group of $M$ is

$$
\Gamma^{k}(M)=\pi_{0}\left(\operatorname{Diff}\left(M, \mathbf{m}_{k}\right)\right),
$$

where $\operatorname{Diff}\left(M, \mathbf{m}_{k}\right)$ is the group of diffeomorphisms from $M$ to itself that fix each point of a given set $\mathbf{m}_{k}$ of $k$ marked points in $M$. If $M$ has boundary, then we insist that the diffeomorphisms restrict to the identity on the boundary; and if $M$ is orientable, the diffeomorphisms must be orientation-preserving. This definition is also meaningful when $k=0$, in which case $k$ is conventionally omitted from the notation. In the case of the mapping class groups, the map $d_{i}: \Gamma^{k+1}(M) \rightarrow \Gamma^{k}(M)$ corresponds to the intuitive notion of forgetting the $i$ th marked point (although again there are indexing subtleties).

Evaluating a diffeomorphism at the points of $\mathbf{m}_{k}$ necessarily gives a configuration of $k$ distinct points of $M$, and so an evaluation map

$$
\operatorname{ev}_{\mathbf{m}_{k}}: \operatorname{Diff}(M) \longrightarrow \operatorname{Conf}_{k}(M),
$$

which is a locally trivial fibration with fiber $\operatorname{Diff}\left(M, \mathbf{m}_{k}\right)$ (see Appendix A.1). The connecting homomorphism $\pi_{1} \rightarrow \pi_{0}$ in the long exact sequence associated to the fibration is thus a natural map $P_{k}(M) \rightarrow \Gamma^{k}(M)$. This homomorphism allows us to compare the structures we impose on these collections of groups:

$$
\begin{aligned}
& \underline{P}(M)=\left\{P_{1}(M), P_{2}(M), \ldots\right\}, \\
& \underline{\Gamma}(M)=\left\{\Gamma^{1}(M), \Gamma^{2}(M), \ldots\right\} .
\end{aligned}
$$

The pure braid groups $P_{k}(M)$ and pure mapping class groups $\Gamma^{k}(M)$ are each subgroups of important larger groups, namely the full braid group

$$
\operatorname{Br}_{k}(M)=\pi_{1}\left(\Sigma_{k} \backslash \operatorname{Conf}_{k}(M)\right)
$$


where $\Sigma_{k} \backslash \operatorname{Conf}_{\mathrm{k}}(\mathrm{M})$ is the quotient of the configuration space by the natural symmetric group action, and the full mapping class group given by

$$
\Gamma^{(k)}(M)=\pi_{0}\left(\operatorname{Diff}\left(M,\left[\mathbf{m}_{k}\right]\right)\right),
$$

where $\operatorname{Diff}\left(M,\left[\mathbf{m}_{k}\right]\right)$ is the same as $\operatorname{Diff}\left(M, \mathbf{m}_{k}\right)$ except that here the diffeomorphisms need only fix the set $\left[\mathbf{m}_{k}\right]$ of marked points. In particular, if $M$ is orientable, the diffeomorphisms must be orientation-preserving (this is our convention whenever we use the notation 'Diff').

It is very natural to ask whether there are also $\Delta$-structures on these full groups. For the sake of completeness, and in order to generalize the work in [1, we consider this below.

Just as in the pure case, there is a natural map $\operatorname{Br}_{k}(M) \rightarrow \Gamma^{(k)}(M)$. This is the connecting homomorphism in the sequence associated to the composite fibration

$$
\operatorname{Diff}(M) \stackrel{\mathrm{ev}_{\mathbf{m}_{k}}}{\longrightarrow} \operatorname{Conf}_{k}(M) \rightarrow \Sigma_{k} \backslash \operatorname{Conf}_{k}(M)
$$

whose fiber is $\operatorname{Diff}\left(M,\left[\mathbf{m}_{k}\right]\right)$.

Unlike in the pure case, the sequences

$$
\begin{aligned}
\underline{\mathrm{Br}}(M) & =\left\{\mathrm{Br}_{1}(M), \mathrm{Br}_{2}(M), \ldots\right\}, \\
\underline{\Gamma}^{\mathrm{sym}}(M) & =\left\{\Gamma^{(1)}(M), \Gamma^{(2)}(M), \ldots\right\}
\end{aligned}
$$

do not form $\Delta$-groups. As above, there are well-defined functions $d_{i}: \operatorname{Br}_{k+1}(M) \rightarrow$ $\operatorname{Br}_{k}(M)$ and $d_{i}: \Gamma^{(k+1)}(M) \rightarrow \Gamma^{(k)}(M)$, also known as face maps, given by deleting the $i$ th strand or forgetting the $i$ th marked point; but these are not group homomorphisms. As first noted by Fiedorowicz-Loday in the case of Artin braids [11. (with further examples due to Connes [7] and Loday [15]), permutations intervene in an essential way. In [1] it is shown that $\underline{\operatorname{Br}}(M)$ is actually a crossed $\Delta$-group, meaning that there are homomorphisms $\pi: \operatorname{Br}_{k+1}(M) \rightarrow \Sigma_{k+1}$ that commute with the face maps and that the identity $d_{i}\left(x_{1} x_{2}\right)=d_{i}\left(x_{1}\right) d_{i \cdot \pi\left(x_{1}\right)}\left(x_{2}\right)$ holds for all $x_{1}, x_{2}$ in $\operatorname{Br}_{k+1}(M)$ and $i=0, \ldots, k$. Here, $i \cdot \pi\left(x_{1}\right)$ denotes the result of performing the permutation $\pi\left(x_{1}\right)$ on $i$. Homological consequences of such structures are developed in [11], whilst applications to the theory of group operads and iterated loop spaces in homotopy theory may be found in 22 .

Our first main result shows that $\underline{\Gamma}(M)$ is also a crossed $\Delta$-group, and explores compatibility with the braid groups, as follows.

Theorem A. (a) The sequence $\underline{\Gamma}^{\mathrm{sym}}(M)=\left\{\Gamma^{(1)}(M), \Gamma^{(2)}(M), \ldots\right\}$ forms a crossed $\Delta$-group.

(b) The natural homomorphism $\operatorname{Br}_{k}(M) \rightarrow \Gamma^{(k)}(M)$ commutes with the face maps and the maps to the symmetric groups; that is, there is a morphism $\underline{\operatorname{Br}}(M) \rightarrow$ $\underline{\Gamma}^{\mathrm{sym}}(M)$ of crossed $\Delta$-groups.

The general theory of $\Delta$-groups allows us to deduce:

Corollary B. (a) The sequence $\underline{\Gamma}(M)=\left\{\Gamma^{1}(M), \Gamma^{2}(M), \ldots\right\}$ forms a $\Delta$-group.

(b) The natural homomorphism $P_{k}(M) \rightarrow \Gamma^{k}(M)$ commutes with the face maps; that is, there is a morphism $\underline{P}(M) \rightarrow \underline{\Gamma}(M)$ of $\Delta$-groups.

We then compare the two $\Delta$-groups $\underline{P}(M)$ and $\underline{\Gamma}(M)$. By using the $\Delta$-structures, we are able to deduce results about two important subgroups, namely the Brunnian braid group $\operatorname{Brun}\left(P_{k+1}(M)\right) \leq P_{k+1}(M)$ and the Brunnian mapping class group 
$\operatorname{Brun}\left(\Gamma^{k+1}(M)\right) \leq \Gamma^{k+1}(M)$. The first of these consists of those braids that become trivial when any one of their strands is removed, and the second consists of mapping classes represented by diffeomorphisms that are isotopic to the identity if any one of the marked points is forgotten.

Theorem C. For any $k \geq 0$ the homotopy sets $\pi_{k}(\underline{P}(M))$ and $\pi_{k}(\underline{\Gamma}(M))$ are groups. There are isomorphisms $\pi_{k}(\underline{P}(M)) \cong \pi_{k}(\underline{\Gamma}(M))$ and $\operatorname{Brun}\left(P_{k+1}(M)\right) \cong$ $\operatorname{Brun}\left(\Gamma^{k+1}(M)\right)$ in degrees

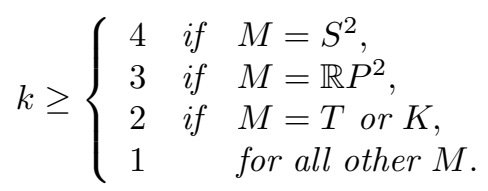

Behaviour in lower degrees is calculated in our second paper [3], which continues the study of $\underline{P}(M)$ and $\underline{\Gamma}(M)$ and uses our present work to deduce further results about the Brunnian braid groups and Brunnian mapping class groups. In particular, it generalizes the exact sequence of [1].

This paper is organized as follows. In Section 2 we recall some facts about crossed $\Delta$-groups and the crossed $\Delta$-group structure on the braid groups (from [1]). Turning to mapping class groups, we prove part (a) of Theorem A and deduce part (a) of Corollary B. Section 3 studies compatibility of the connecting homomorphism

$$
\operatorname{Br}_{k}(M) \rightarrow \Gamma^{(k)}(M)
$$

with the crossed $\Delta$-group structures, leading to a proof of part (b) of Theorem A and part (b) of Corollary B. In the pure case, this corresponds to the vertical faces of a large commuting diagram (3.2). Its horizontal faces are explored in the second paper, which addresses the kernels of face maps in order to study the Brunnian groups that arise. Then, in Section 4, we explain the definition of the homotopy sets of a $\Delta$-group, and prove Theorem C. Finally, the Appendix presents rigorous proofs of a number of intuitively feasible results that occur in the main text and which, often for notational reasons, are technically more complicated than one might expect.

\section{Establishing the $\Delta$-STRUCtures}

A $\Delta$-set is a sequence of sets $\underline{X}=\left\{X_{0}, X_{1}, \ldots\right\}$ together with face maps $d_{i}$ : $X_{k} \rightarrow X_{k-1}$ for each $i=0, \ldots, k$ that satisfy the face map identity

$$
d_{j} d_{i}=d_{i} d_{j+1} \text { whenever } i \leq j .
$$

If each $X_{k}$ is a group and the face maps are group homomorphisms, then $\underline{X}$ is said to be a $\Delta$-group.

2.1. The $\Delta$-structure on the symmetric groups. The collection of symmetric groups $\underline{\Sigma}=\left\{\Sigma_{k+1}\right\}_{k \geq 0}$ is an important example of a $\Delta$-set. We regard $\Sigma_{k}$ as the group of permutations of $[k]=\{0, \ldots, k-1\}$ and write $i \cdot \sigma$ for the result of evaluating $\sigma$ on $i$. Thus $\sigma \tau$ denotes the permutation obtained by first applying $\sigma$ and then $\tau$. 


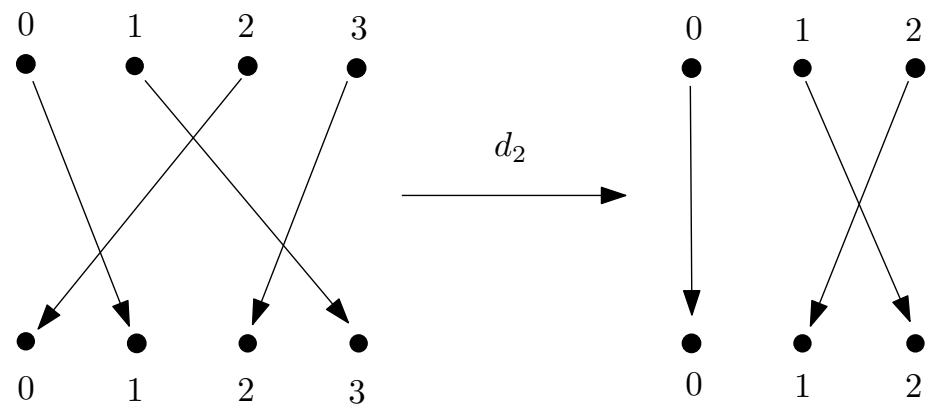

FiguRE 1. The face maps for the symmetric groups

The face maps $d_{i}: \Sigma_{k} \rightarrow \Sigma_{k-1}(i=0,1, \ldots, k-1)$ are defined by commutative diagrams

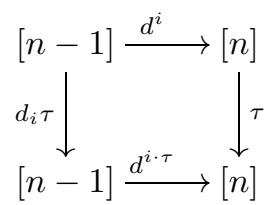

where $d^{i}:[n-1] \rightarrow[n]$ is the unique order-preserving map that misses $i$. If we represent a permutation by a diagram of arrows as in Figure 1, then $d_{i}$ removes the arrow that starts at $i$. It can easily be checked that the face map identity holds in this case. Note that $d_{i}$ is not a group homomorphism, so we obtain only a $\Delta$-set.

For future reference, we record the following result about the behaviour of the face maps. We recall that a pair $i<j$ with $i, j \in\{1, \ldots, k-1\}$ is said to be inverted by a permutation $\sigma$ if $i \cdot \sigma>j \cdot \sigma$. Evidently, a permutation is trivial if and only if it does not invert any pairs. Recall also that $\Sigma_{k}$ is generated by transpositions $\tau_{j}=(j j+1)$ for $0 \leq j<k-1$.

Lemma 2.1. Let $\mathrm{K}_{r}\left(\Sigma_{k}\right)=\operatorname{Ker} d_{r} \subseteq \Sigma_{k}$ denote the set of permutations mapped to the trivial permutation by $d_{r}$. The face maps on $\Sigma_{k}$ have the following properties.

(a) For $0 \leq r \leq k-1, \mathrm{~K}_{r}\left(\Sigma_{k}\right)$ comprises those $\sigma \in \Sigma_{k}$ with the property that for any pair $i<j$ inverted by $\sigma$, we have $r \in\{i, j\}$.

(b) For $0 \leq r<s \leq k-1$,

$$
\mathrm{K}_{r}\left(\Sigma_{k}\right) \cap \mathrm{K}_{s}\left(\Sigma_{k}\right)=\left\{\begin{array}{cl}
\left\langle\tau_{r}\right\rangle \cong \Sigma_{2} & \text { if } s=r+1, \\
1 & \text { otherwise. }
\end{array}\right.
$$

(c) For $0 \leq r<s<t \leq k-1$,

$$
\mathrm{K}_{r}\left(\Sigma_{k}\right) \cap \mathrm{K}_{s}\left(\Sigma_{k}\right) \cap \mathrm{K}_{t}\left(\Sigma_{k}\right)=1 .
$$

Proof. (a) By (2.1), the pair $i<j$ is inverted by $d_{r}(\sigma)$ if and only if

$$
d^{r}(i) \cdot \sigma=d^{r \cdot \sigma}\left(i \cdot d_{r}(\sigma)\right)>d^{r \cdot \sigma}\left(j \cdot d_{r}(\sigma)\right)=d^{r}(j) \cdot \sigma ;
$$

that is, if and only if the pair $d^{r}(i)<d^{r}(j)$ is inverted by $\sigma$. By the definition of $d^{r}$, neither $d^{r}(i)$ nor $d^{r}(j)$ equals $r$.

Now suppose that $\sigma \in \mathrm{K}_{r}\left(\Sigma_{k}\right)$, and $i^{\prime}<j^{\prime}$ is inverted by $\sigma$; then one of $i^{\prime}, j^{\prime}$ must be equal to $r$. For if not, we have $i^{\prime}=d^{r}(i), j^{\prime}=d^{r}(j)$ for some $0 \leq i<j \leq k-2$; and so $i<j$ is inverted by $d_{r}(\sigma)$, giving a contradiction. 
Conversely, if every pair inverted by $\sigma$ contains $r$, then none of them is of the form $d^{r}(i)<d^{r}(j)$; so, there are no pairs $i<j$ inverted by $d_{r}(\sigma)$, i.e. $d_{r}(\sigma)$ is trivial.

(b) If $\sigma$ is a nontrivial element of $\mathrm{K}_{r}\left(\Sigma_{k}\right) \cap \mathrm{K}_{s}\left(\Sigma_{k}\right)$, then by (a) the unique pair inverted by $\sigma$ is $r<s$. Since there is only one pair inverted, we must have $s=r+1$.

(c) This is now immediate.

2.2. The definition of a crossed $\Delta$-group. There is an intermediate notion between a $\Delta$-set and a $\Delta$-group, that of a crossed $\Delta$-group. A crossed $\Delta$-group is a $\Delta$-set $\underline{X}=\left\{X_{k}\right\}_{k \geq 0}$ for which each $X_{i}$ is a group and there are homomorphisms $\pi: X_{k} \rightarrow \Sigma_{k+1}$ that commute with the face maps and satisfy

$$
d_{i}\left(x_{1} x_{2}\right)=d_{i}\left(x_{1}\right) d_{i \cdot \pi\left(x_{1}\right)}\left(x_{2}\right) .
$$

(Note that there are several choices to be made with regards to which side the symmetric group acts on $\{0,1, \ldots, k-1\}$ and how the face maps for symmetric groups are defined. Different choices give rise to different versions of this identity.)

The symmetric groups with $\pi$ as the identity map form the prototypical crossed $\Delta$-group. If $\underline{X}$ is any other crossed $\Delta$-group, then the homomorphisms $\pi: X_{k} \rightarrow$ $\Sigma_{k+1}$ fit together to give a morphism of crossed $\Delta$-groups $\pi: \underline{X} \rightarrow \underline{\Sigma}$, that is, a collection of maps respecting the crossed $\Delta$-group structure.

Any $\Delta$-group is a crossed $\Delta$-group with $\pi$ taken to be the trivial homomorphism. In the converse direction, a crossed $\Delta$-group need not be a $\Delta$-group.

For a $\Delta$-group $\underline{X}$ we define

$$
\mathrm{K}_{i}\left(X_{k}\right)=\operatorname{Ker}\left[d_{i}: X_{k} \rightarrow X_{k-1}\right] .
$$

Since $\pi$ commutes with the face maps, the following is immediate from Lemma 2.1 .

Proposition 2.2. Let $\underline{X}=\left\{X_{k}\right\}_{k \geq 0}$ be a crossed $\Delta$-group with associated homomorphisms $\pi: X_{k} \rightarrow \Sigma_{k+1}$. If $i_{1}, i_{2}, i_{3} \in\{0, \ldots, k\}$ are all distinct, then

$$
\mathrm{K}_{i_{1}}\left(X_{k}\right) \cap \mathrm{K}_{i_{2}}\left(X_{k}\right) \cap \mathrm{K}_{i_{3}}\left(X_{k}\right) \leq \operatorname{Ker} \pi .
$$

Another easily checked, useful fact about crossed $\Delta$-groups is as follows.

Lemma 2.3. If $\underline{X}$ is a crossed $\Delta$-group and $\pi: \underline{X} \rightarrow \underline{\Sigma}$ is the associated morphism, then $\operatorname{Ker} \pi \leq \underline{X}$ is a $\Delta$-group.

2.3. The $\Delta$-structure on braid groups. In this section it is convenient to use an alternative description of the braid groups. Let $B_{k}(M)$ denote the set of pathhomotopy classes $[\lambda]$ of paths $\lambda$ in $\operatorname{Conf}_{k}(M)$ that start at the base configuration $\mathbf{m}_{k}$ and end at some permutation of it. This is the description appearing in [1].

To define the multiplication on $B_{k}(M)$, we use the map

$$
\pi: B_{k}(M) \rightarrow \Sigma_{k}
$$

that sends $[\lambda] \in B_{k}(M)$ to the permutation $\pi[\lambda]$ defined by

$$
\lambda(1)=\left(m_{0 \cdot \pi[\lambda]}, \ldots, m_{(k-1) \cdot \pi[\lambda]}\right) .
$$

The kernel of $\pi$ is $P_{k}(M)=\pi_{1}\left(\operatorname{Conf}_{k}(M), \mathbf{m}_{k}\right)$.

For $[\lambda] \in B_{k}(M)$, let $\left(\lambda^{0}, \ldots, \lambda^{k-1}\right)$ be the coordinates of $\lambda$. There is a left action of $\Sigma_{k}$ on $\operatorname{Conf}_{k}(M)$ given by

$$
\sigma \cdot\left(x_{0}, \ldots, x_{k-1}\right)=\left(x_{0 \cdot \sigma}, \ldots, x_{(k-1) \cdot \sigma}\right)
$$


and this induces a left action of $\Sigma_{k}$ on $B_{k}(M)$ given by

$$
\sigma \cdot[\lambda]=[\sigma \cdot \lambda]
$$

where $\sigma \cdot \lambda$ denotes the path $\left(\lambda^{0 \cdot \sigma}, \ldots, \lambda^{k \cdot \sigma}\right)$. Now the product in $B_{k}(M)$, which we denote by $\circledast$, is given by

$$
\left[\lambda_{1}\right] \circledast\left[\lambda_{2}\right]=\left[\lambda_{1} *\left(\pi\left(\lambda_{1}\right) \cdot \lambda_{2}\right)\right],
$$

where $*$ denotes the usual concatenation product of paths (read from left to right). With this multiplication, the map $\pi$ is a group homomorphism.

This group is isomorphic to the group $\operatorname{Br}_{k}(M)$ that we originally took to be the braid group, as follows. The quotient map $p: \operatorname{Conf}_{k}(M) \rightarrow \Sigma_{k} \backslash \operatorname{Conf}_{k}(M)$ is a covering map. Thus, if we have a loop $\gamma$ in $\Sigma_{k} \backslash \operatorname{Conf}_{k}(M)$ based at $\left[\mathbf{m}_{k}\right]$, then there exists a unique lift of $\gamma$ to a path $\tilde{\gamma}$ in $\operatorname{Conf}_{k}(M)$ starting at $\mathbf{m}_{k}$. This establishes the isomorphism

$$
g: \operatorname{Br}_{k}(M)=\pi_{1}\left(\Sigma_{k} \backslash \operatorname{Conf}_{k}(M),\left[\mathbf{m}_{k}\right]\right) \stackrel{\cong}{\longrightarrow} B_{k}(M) .
$$

In 1 it is shown that for any surface $M$, the braid groups of $M$ form a crossed $\Delta$-group $\underline{B}(M)$ with

$$
\underline{B}(M)_{k}=B_{k+1}(M) .
$$

The crossed $\Delta$-group structure is defined as follows. First, the crossed homomorphism $\underline{B}(M)_{k} \rightarrow \Sigma_{k+1}$ is provided by the homomorphism $\pi$ defined above.

The face map $d_{i}: B_{k+1}(M) \rightarrow B_{k}(M)$ removes the $i$ th component of a path in $\operatorname{Conf}_{k+1}(M)$. Geometrically, we think of $d_{i}$ as removing the $i$ th strand of a braid, as in Figure 2. A slight modification is necessary so that the resulting path represents an element of $B_{k}(M)$. We now explain the details.

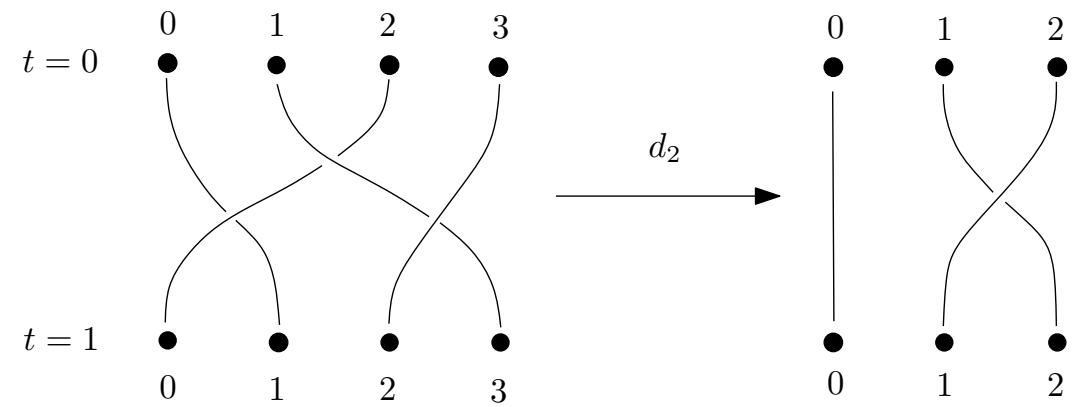

Figure 2. Deleting a strand

To make a good selection of the marked points $\mathbf{m}_{k}$ we employ the notion of a whisker in the surface $M$. This is a smooth embedding $\rho: \mathbb{R} \rightarrow M-\partial M$ with the property that the closure of $\operatorname{Im} \rho$ is homeomorphic to an interval. The chosen whisker $\rho$ in $M$ determines the marked points in $M$ : for $i=0,1, \ldots$ (and more generally for any $i \in \mathbb{Z})$ define $m_{i}=\rho(i)$ and then $\mathbf{m}_{k}=\left(m_{0}, \ldots, m_{k-1}\right) \in$ $\operatorname{Conf}_{k}(M)$. We write $\mathbf{m}_{k}^{i}$ for the sequence of marked points $\left(m_{0}, \ldots, \hat{m}_{i}, \ldots, m_{k-1}\right)$.

To define the face map $d_{i}: B_{k+1}(M) \rightarrow B_{k}(M)$ precisely, we use a path $\delta_{k, i}$ : $I \rightarrow \operatorname{Conf}_{k}(M)$ that begins at $\mathbf{m}_{k}$ and ends at $\mathbf{m}_{k+1}^{i}$. The path slides the marked points along the whisker; an explicit description is given in Appendix A.2. 


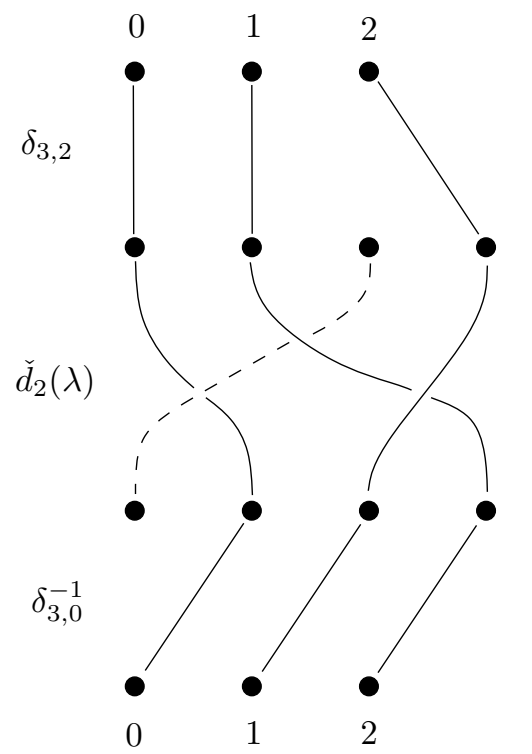

Figure $3 . d_{2}(\lambda)$ for the braid $\lambda$ in Figure 2

If $\lambda=\left(\lambda^{0}, \ldots, \lambda^{k}\right)$ is a path in $\operatorname{Conf}_{k+1}(M)$, write $\lambda^{\mathrm{I}}$ for its inverse path, and $\check{d}_{i} \lambda$ for the path $\left(\lambda^{0}, \ldots, \hat{\lambda}^{i}, \ldots, \lambda^{k}\right)$ in $\operatorname{Conf}_{k}(M)$. Then, for $i=0, \ldots, k$, put

$$
d_{i} \lambda=\delta_{k, i} * \check{d}_{i} \lambda *\left(d_{i} \pi(\lambda) \cdot \delta_{k, i \cdot \pi(\lambda)}^{\mathrm{I}}\right),
$$

and finally define $d_{i}[\lambda]=\left[d_{i} \lambda\right] \in B_{k}(M)$; see Figure 3.

Theorem 2.4 ([1, Proposition 4.2.1 (1)]). For an arbitrary surface $M$, the collection $\underline{B}(M)=\left\{B_{k+1}(M)\right\}_{k \geq 0}$ forms a crossed $\Delta$-group.

Using the isomorphisms $g: \operatorname{Br}_{k}(M) \stackrel{\cong}{\rightrightarrows} B_{k}(M)$, the theorem gives us a crossed $\Delta$-group structure on $\underline{\mathrm{Br}}(M)$ in which the maps to the symmetric groups are given by $\pi \circ g$.

The kernel of $\pi: \underline{B}(M) \rightarrow \underline{\Sigma}$ is $\underline{P}(M)$, where

$$
\underline{P}(M)_{k}=P_{k+1}(M),
$$

the $(k+1)$ st pure braid group of $M$. Thus, from Lemma 2.3 we immediately have the following.

Corollary 2.5 ([1, Proposition 4.2.1 (2)]). For an arbitrary surface $M$, the collection $\underline{P}(M)=\left\{P_{k+1}(M)\right\}_{k \geq 0}$ forms a $\Delta$-group.

Further, the following application of Proposition 2.2 shows how pure braids commonly arise.

Proposition 2.6. Any braid that lies in the kernels of three distinct face maps is a pure braid.

An extension of Theorem 2.4 and Corollary 2.5 occurs in [1], where it is shown that when the surface $M$ admits a nonvanishing vector field, then the pure (respectively, full) braid groups may be endowed with degeneracy maps as well as face maps, and therefore form a simplicial group (resp., a crossed simplicial group). 
2.4. The $\Delta$-structure on mapping class groups. In this section we show that $\underline{\Gamma}^{\text {sym }}(M)$ defined by

$$
\underline{\Gamma}^{\mathrm{sym}}(M)_{k}=\Gamma^{(k+1)}(M)
$$

is a crossed $\Delta$-group and $\underline{\Gamma}(M)$ given by

$$
\underline{\Gamma}(M)_{k}=\Gamma^{k+1}(M)
$$

is a $\Delta$-group. The superscript 'sym' is intended to remind the reader that these mapping class groups admit nontrivial homomorphisms to the symmetric groups.

We use $x \cdot \phi$ (respectively $\mathbf{x} \cdot \phi)$ to denote the result of evaluating a diffeomorphism $\phi$ on the point $x \in M$ (resp. $\left.\mathbf{x} \in \operatorname{Conf}_{k+1}(M)\right)$ and $\phi \odot \psi$ to denote the diffeomorphism given by first performing $\phi$ and then $\psi$. The right action of the diffeomorphism group on the surface is thereby compatible with our other choices of actions. Specifically, the following holds.

Lemma 2.7. The actions of a permutation $\sigma$ and diffeomorphism $\phi$ on a configuration $\mathbf{x} \in \operatorname{Conf}_{k+1}(M)$ are compatible, in that

$$
(\sigma \cdot \mathbf{x}) \cdot \phi=\sigma \cdot(\mathbf{x} \cdot \phi)
$$

Proof. For $i=0, \ldots, k$, write $y_{i}=x_{i} \cdot \phi$. Then each configuration in the asserted equation is equal to $\left(y_{0 \cdot \sigma}, \ldots, y_{k \cdot \sigma}\right)$.

Define $\mu: \Gamma^{(k+1)}(M) \rightarrow \Sigma_{k+1}$ by

$$
\mathbf{m}_{k+1} \cdot \psi=\mu[\psi] \cdot \mathbf{m}_{k+1}
$$

for $[\psi]$ in $\Gamma^{(k+1)}(M)$. Thus, $\mu$ records how the diffeomorphisms permute the marked points. We also use the notation $\mu$ for the composite

$$
\operatorname{Diff}\left(M,\left[\mathbf{m}_{k+1}\right]\right) \longrightarrow \pi_{0}\left(\operatorname{Diff}\left(M,\left[\mathbf{m}_{k+1}\right]\right)\right)=\Gamma^{(k+1)}(M) \stackrel{\mu}{\longrightarrow} \Sigma_{k+1} .
$$

It is clear from the definition that $\mu$ is a group homomorphism; an explicit proof may be written using the previous lemma.

Naively speaking, the face map $d_{i}: \Gamma^{(k+1)}(M) \rightarrow \Gamma^{(k)}(M)$ forgets the $i$ th marked point. However, if we simply forget the marked points, then $d_{i} \phi$ and $d_{j} \phi$ would lie in different groups - the first in the group of diffeomorphisms that fix $\mathbf{m}_{k}^{i}$ and the second in the group of diffeomorphisms that fix $\mathbf{m}_{k}^{j}$.

To rectify this, we use a diffeomorphism $\theta_{i}: M \rightarrow M$ for each $i=0,1, \ldots$ The diffeomorphism $\theta_{i}$ is supported in a neighbourhood of the marked points and whenever $i \leq k$ carries $\mathbf{m}_{k}$ onto $\mathbf{m}_{k+1}^{i}$ by moving the points along the whisker. In particular, $\theta_{i}$ preserves the order of the points. An explicit construction of $\theta_{i}$ can be found in Appendix A.2.

The face map $d_{i}: \Gamma^{(k+1)}(M) \rightarrow \Gamma^{(k)}(M)$ is given by

$$
d_{i}[\phi]=\left[\theta_{i} \odot \phi \odot \theta_{i \cdot \mu(\phi)}^{-1}\right] .
$$

In other words, we have a commutative diagram

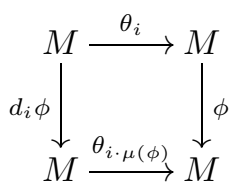


To check that $d_{i} \phi$ preserves the set $\left\{m_{0}, \ldots, m_{k-1}\right\}$, observe that this commutative diagram restricts to the following diagram of bijections:

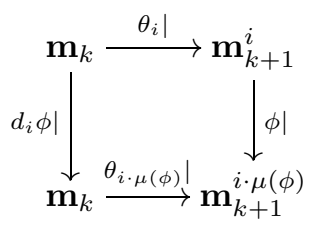

It is intuitively clear that the face maps satisfy the face map identity, i.e. that $d_{j} d_{i}=d_{i} d_{j+1}$ whenever $i \leq j$. Forgetting the $i$ th marked point and then the $j$ th marked point is exactly the same as forgetting the $(j+1)$ st marked point and then the $i$ th marked point. This gives the next result; a rigorous proof can be found in Appendix A.4

Proposition 2.8. The face maps $d_{i}: \Gamma^{(k+1)}(M) \rightarrow \Gamma^{(k)}(M)$ defined above satisfy the face map identity.

Theorem 2.9. For any surface $M$, the collection

$$
\underline{\Gamma}^{\mathrm{sym}}(M)=\left\{\Gamma^{(k+1)}(M)\right\}_{k \geq 0}
$$

forms a crossed $\Delta$-group.

Proof. Given the preceding proposition, it suffices to check that the map $\mu$ : $\Gamma^{(k+1)}(M) \rightarrow \Sigma_{k+1}$ both (a) commutes with the face maps, and (b) satisfies the crossed identity.

(a) By the definition of the face maps for symmetric groups, in order to see that $d_{i}(\mu(\phi))=\mu\left(d_{i}(\phi)\right)$, it is enough to check that $\mu\left(d_{i}(\phi)\right)$ fits into a commutative diagram

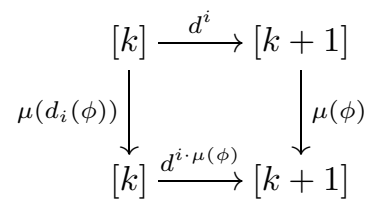

This diagram is isomorphic to the diagram

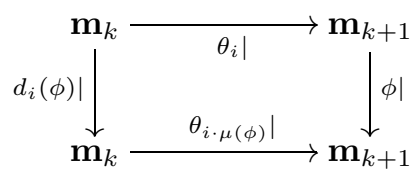

which is commutative by the definition of the face maps for mapping class groups.

(b) We check that the crossed identity holds, i.e. that for all $\phi_{1}, \phi_{2} \in \Gamma^{(k+1)}(M)$ and for all $i=0, \ldots, k$,

$$
d_{i}\left(\phi_{1} \odot \phi_{2}\right)=d_{i}\left(\phi_{1}\right) \odot d_{i \cdot \mu\left(\phi_{1}\right)}\left(\phi_{2}\right) .
$$

To see this, observe that $d_{i}\left(\phi_{1}\right) \odot d_{i \cdot \mu\left(\phi_{1}\right)}\left(\phi_{2}\right)$ is equal to

$$
\begin{aligned}
& \theta_{i} \odot \phi_{1} \odot \theta_{i \cdot \mu\left(\phi_{1}\right)}^{-1} \odot \theta_{i \cdot \mu\left(\phi_{1}\right)} \odot \phi_{2} \odot \theta_{\left(i \cdot \mu\left(\phi_{1}\right)\right) \cdot \mu\left(\phi_{2}\right)} \\
& =\theta_{i} \odot\left(\phi_{1} \odot \phi_{2}\right) \odot \theta_{i \cdot\left(\mu\left(\phi_{1}\right) \mu\left(\phi_{2}\right)\right)} \\
& =\theta_{i} \odot\left(\phi_{1} \odot \phi_{2}\right) \odot \theta_{i \cdot \mu\left(\phi_{1} \odot \phi_{2}\right)} \\
& =d_{i}\left(\phi_{1} \odot \phi_{2}\right) .
\end{aligned}
$$


The kernel of the morphism $\mu: \underline{\Gamma}^{\mathrm{sym}}(M) \rightarrow \underline{\Sigma}$ is $\underline{\Gamma}(M)$. Using Lemma 2.3, this implies the following result.

Corollary 2.10. For any surface $M$, the collection $\underline{\Gamma}(M)=\left\{\Gamma^{k+1}(M)\right\}_{k \geq 0}$ forms a $\Delta$-group.

We end with the following immediate application of Proposition 2.2

Proposition 2.11. If an element in $\Gamma^{(k+1)}(M)$ lies in the kernels of three distinct face maps, then it does not permute the marked points, i.e. it lies in $\Gamma^{k+1}(M)$.

In 2 there is an extension of Theorem 2.9 and Corollary 2.10, whereby it is shown that when the surface $M$ has nonempty boundary, then the pure (respectively, full) mapping class groups admit degeneracy maps as well as face maps, and therefore form a simplicial group (resp., crossed simplicial group).

\section{Compatibility of the $\Delta$-Structures FOR BRAID AND MAPPING CLASS GROUPS}

Let us begin with an intuitive description of why the $\Delta$-structures for braid groups and mapping class groups are compatible. We begin by recalling the map from braid groups to mapping class groups. The evaluation map

$$
\operatorname{ev}_{\mathbf{m}_{k}}: \operatorname{Diff}(M) \longrightarrow \operatorname{Conf}_{k}(M)
$$

is a fibration with fiber $\operatorname{Diff}\left(M, \mathbf{m}_{k}\right)$, as is shown in Appendix A.1. If we compose this with the covering map

$$
p: \operatorname{Conf}_{k}(M) \longrightarrow \Sigma_{k} \backslash \operatorname{Conf}_{k}(M),
$$

we obtain a fibration $p \circ \mathrm{ev}_{\mathbf{m}_{k}}$ with fiber $\operatorname{Diff}\left(M,\left[\mathbf{m}_{k}\right]\right)$. This fibration gives rise to the long exact sequence

$$
\cdots \longrightarrow \pi_{1}(\operatorname{Diff}(M)) \longrightarrow \operatorname{Br}_{k}(M) \stackrel{\partial}{\longrightarrow} \Gamma^{(k)}(M) \longrightarrow \Gamma(M) \rightarrow 1 .
$$

We can describe the connecting homomorphism as follows. Suppose that we are given a loop $\alpha: I \rightarrow \Sigma_{k} \backslash \operatorname{Conf}_{k}(M)$ based at $\left[\mathbf{m}_{k}\right]$ representing an element $[\alpha] \in \operatorname{Br}_{k}(M)$. We may think of $\alpha$ as a path in $\operatorname{Conf}_{k}(M)$ that starts at $\mathbf{m}_{k}$ and ends at some permutation of it. To calculate $\partial[\alpha]$ we must find a lift of $\alpha$ to a path $\Phi_{\alpha}: I \rightarrow \operatorname{Diff}(M)$ that starts at the identity, and then $\partial[\alpha]=\Phi_{\alpha}(1)$.

For the path $\Phi_{\alpha}$ we may choose the diffeotopy of $M$ given by pushing a small neighbourhood of $m_{i}$ around the path $\alpha^{i}$, the $i$ th component of $\alpha$, for $i=0, \ldots, k$. Intuitively, we may think of putting one finger on each $m_{i}$ and pushing it around $\alpha^{i}$. More precisely, this may be described as the diffeotopy extension of the isotopy $\mathbf{m}_{k} \times I \rightarrow M$ defined by $\alpha$, which exists by e.g. [12, Chapter 8 , Theorem 1.3.

From this description we see that forgetting the $i$ th path in $\alpha$ is the same as forgetting the $i$ th point in $\partial[\alpha]$. Moreover, $\partial$ gives rise to a commuting triangle associated to the maps from $\operatorname{Br}_{k}(M)$ and $\Gamma^{(k)}(M)$ to the symmetric group.

A rigorous proof of compatibility in the case of the full braid group and full mapping class group is provided in Appendix A.3. In this section, we investigate the compatibility in the pure case by proving the commutativity of the following diagram which has exact rows. We fix $k \geq 1$, and suppose that $0 \leq j \leq k-1$. 
As ever, $M$ is a connected surface, with or without boundary and not necessarily orientable.

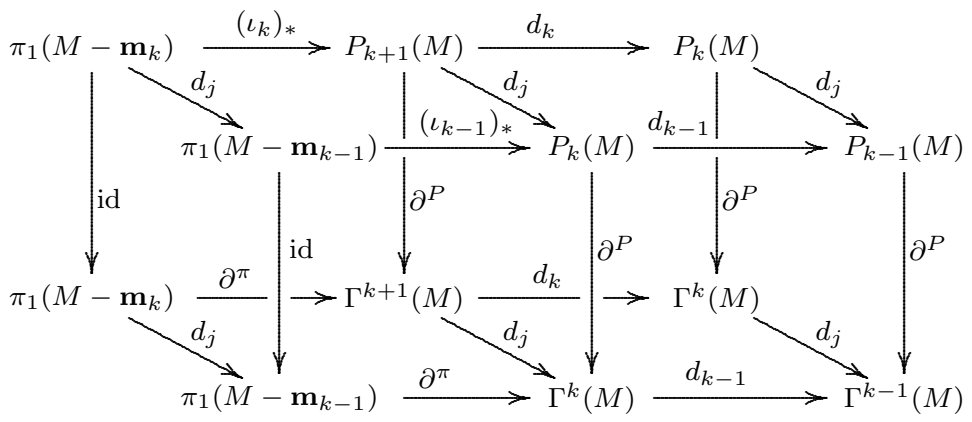

This diagram serves as an organizing principle for this paper and its sequel. Because, in order to study Brunnian groups, the second paper focuses on identifying the kernels of face maps, it relates to the horizontal faces of the diagram. Here, compatibility of $\Delta$-structures corresponds to commutativity of the vertical faces.

1. The upper horizontal face. Recall that we use $\mathbf{m}_{k}^{j}$ to denote $\left(m_{0}, \ldots, \hat{m}_{j}, \ldots\right.$, $\left.m_{k-1}\right) \in \operatorname{Conf}_{k}(M)$. The homomorphism

$$
d_{j}^{\pi}: \pi_{1}\left(M-\mathbf{m}_{k}, m_{k}\right) \longrightarrow \pi_{1}\left(M-\mathbf{m}_{k-1}, m_{k-1}\right)
$$

is induced from the inclusion inc : $M-\mathbf{m}_{k} \hookrightarrow M-\mathbf{m}_{k}^{j}$ that fills in the puncture at $m_{j}$. This occurs by the identification of $M-\mathbf{m}_{k}^{j}$ with $M-\mathbf{m}_{k-1}$ via the diffeomorphism $\theta_{j}:\left(M, \mathbf{m}_{k-1}\right) \rightarrow\left(M, \mathbf{m}_{k}^{j}\right)$ that was introduced in Section 2.4 above. Also, we define a map $\iota_{k}: M-\mathbf{m}_{k} \rightarrow \operatorname{Conf}_{k+1}(M)$ given by $x \mapsto\left(m_{0}, \ldots, m_{k-1}, x\right)$, which induces on fundamental groups the homomorphism

$$
\left(\iota_{k}\right)_{*}: \pi_{1}\left(M-\mathbf{m}_{k}, m_{k}\right) \longrightarrow P_{k+1}(M) .
$$

As a convention, we take $\operatorname{Conf}_{0}(M)$ to consist of a single point and we define $\mathbf{m}_{1}^{0}$ to be the set consisting of that point alone.

Let $\check{d}_{j}: \operatorname{Conf}_{k+1}(M) \rightarrow \operatorname{Conf}_{k}(M)$ be the map given by deleting the point indexed by $j$. Define $\iota_{k}^{j}: M-\mathbf{m}_{k}^{j} \rightarrow \operatorname{Conf}_{k}(M)$ by

$$
\iota_{k}^{j}(x)=\left(m_{0}, \ldots, \hat{m}_{j}, \ldots, m_{k-1}, x\right) .
$$

We have the following commutative diagram of fibrations (in which we have recorded the chosen basepoints, and write $\mathrm{C}_{k}$ for $\left.\operatorname{Conf}_{k}(M)\right)$ :

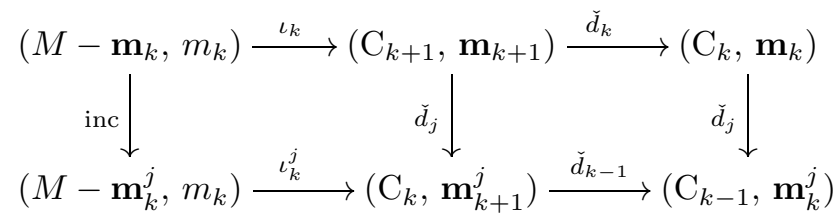

Its associated diagram of exact homotopy sequences includes

$$
\begin{aligned}
& \pi_{1}\left(M-\mathbf{m}_{k}, m_{k}\right) \stackrel{\left(\iota_{k}\right) *}{\longrightarrow} P_{k+1}(M) \stackrel{d_{k}}{\longrightarrow} P_{k}(M) \\
& \text { inc }_{*} \downarrow \\
& \pi_{1}\left(M-\mathbf{m}_{k}^{j}, m_{k}\right) \stackrel{\left(\iota_{k}^{j}\right)_{*}}{\longrightarrow} \pi_{1}\left(\mathrm{C}_{k}, \mathbf{m}_{k+1}^{j}\right) \stackrel{\left(\check{d}_{j}\right)_{*} \downarrow}{\stackrel{\left(\check{d}_{k-1}\right)_{*}}{\longrightarrow}} \pi_{1}\left(\mathrm{C}_{k-1}, \mathbf{m}_{k}^{j}\right)
\end{aligned}
$$


This diagram reduces to the upper horizontal face of (3.2) once we have adjusted the basepoints, as follows. Let $\theta_{j}: M \rightarrow M$ be as above, and, as in Section 2.3 . let $\delta_{k, j}$ be a canonical path from $\mathbf{m}_{k}$ to $\mathbf{m}_{k+1}^{j}$. We use $\operatorname{conj}\left(\delta_{k, j}\right)$ to denote the homomorphism sending the class of a loop $\gamma$ based at $\mathbf{m}_{k+1}^{j}$ to the class of the loop $\delta_{k, j} * \gamma * \delta_{k, j}^{\mathrm{I}}$ based at $\mathbf{m}_{k}$. Commutativity of the following diagram follows from Lemma A.4 ((a) and (c) for the left-hand square, and (d) for the right-hand square):

$$
\begin{gathered}
\pi_{1}\left(M-\mathbf{m}_{k}^{j}, m_{k}\right) \stackrel{\left(\iota_{k}^{j}\right)_{*}}{\longrightarrow} \pi_{1}\left(\mathrm{C}_{k}, \mathbf{m}_{k+1}^{j}\right) \stackrel{\left(\check{d}_{k-1}\right)_{*}}{\longrightarrow} \pi_{1}\left(\mathrm{C}_{k-1}, \mathbf{m}_{k}^{j}\right) \\
\stackrel{\downarrow}{\simeq\left(\theta_{j}^{-1}\right)_{*}} \quad \cong \mid \operatorname{conj}\left(\delta_{k, j}\right) \quad \cong \operatorname{conj}\left(\delta_{k-1, j}\right) \\
\pi_{1}\left(M-\mathbf{m}_{k-1}, m_{k-1}\right) \stackrel{\left(\iota_{k-1}\right)_{*}}{\longrightarrow} P_{k}(M) \stackrel{d_{k-1}^{P}}{\longrightarrow} P_{k-1}(M)
\end{gathered}
$$

The composite of these two commutative diagrams is the required one.

2. The lower horizontal face. The starting point is the diagram of fibrations below. When $k=1$, we interpret $\operatorname{Diff}\left(M, \mathbf{m}_{1}^{0}\right)$ to be the group of diffeomorphisms that are required only to fix $\partial M$. In this case, $\Gamma^{k}(M)=\Gamma(M)$ is the group of isotopy classes of such diffeomorphisms.

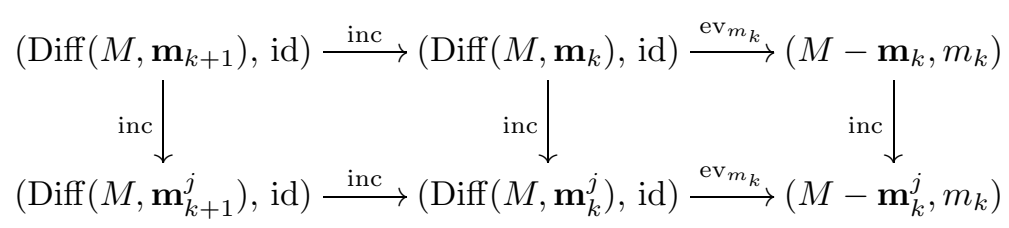

Here $\mathrm{ev}_{m_{k}}$ is given by evaluating a diffeomorphism at the point $m_{k}$. Using $\partial^{\pi}$ to denote the connecting homomorphism, part of the associated diagram of long exact sequences is

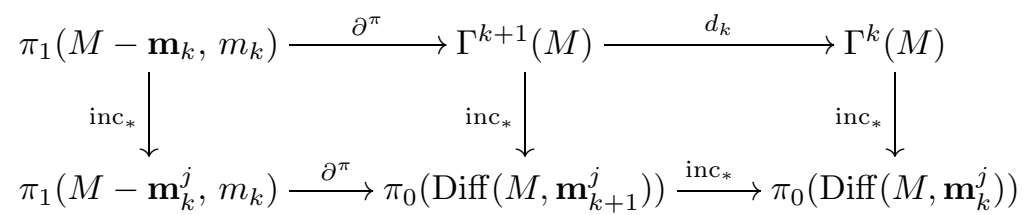

We can describe the connecting homomorphism $\partial^{\pi}: \pi_{1}\left(M-\mathbf{m}_{k}, m_{k}\right) \rightarrow \Gamma^{k+1}(M)$ explicitly. Suppose that we are given a loop $\alpha: I \rightarrow M-\mathbf{m}_{k}$ that represents an element $[\alpha]$ of $\pi_{1}\left(M-\mathbf{m}_{k}, m_{k}\right)$. To calculate $\partial^{\pi}([\alpha])$ we find a lift of $\alpha$ to a path $\Phi_{\alpha}: I \rightarrow \operatorname{Diff}\left(M, \mathbf{m}_{k}\right)$ starting at the identity, and then

$$
\partial^{\pi}([\alpha])=\left[\Phi_{\alpha}(1)\right] \in \pi_{0}\left(\operatorname{Diff}\left(M, \mathbf{m}_{k+1}\right)\right)=\Gamma^{k+1}(M) .
$$

For the path $\Phi_{\alpha}$ we choose the diffeotopy given by pushing a small disc containing the point $m_{k}$ around the loop $\alpha$. This may be described as a diffeotopy extension of the isotopy $\left\{m_{k}\right\} \times I \rightarrow M$ defined by $\alpha$, which exists by e.g. 12 Chapter 8 , Theorem 1.3.

This description of $\partial^{\pi}$ gives commutativity of the left-hand square in the following diagram, in which $\operatorname{conj}\left(\theta_{j}\right)$ sends $[\phi] \in \pi_{0}\left(\operatorname{Diff}\left(M, \mathbf{m}_{k+1}^{j}\right)\right)$ to $\left[\theta_{j} \odot \phi \odot\right.$ $\left.\theta_{j}^{-1}\right] \in \Gamma^{k}(M)$. Commutativity of the right-hand square follows from the observa- 
tion that $d_{k-1}: \Gamma^{k}(M) \rightarrow \Gamma^{k-1}(M)$ is induced by the inclusion $\operatorname{Diff}\left(M, \mathbf{m}_{k}\right) \rightarrow$ $\operatorname{Diff}\left(M, \mathbf{m}_{k-1}\right)$.

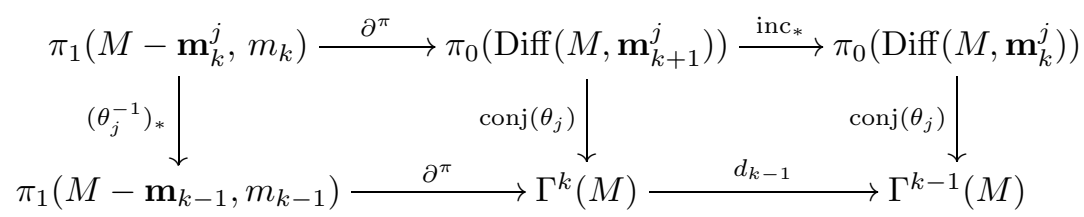

Combining these two diagrams gives the lower horizontal face of (3.2).

3. The vertical faces. There is a commutative diagram of fibrations

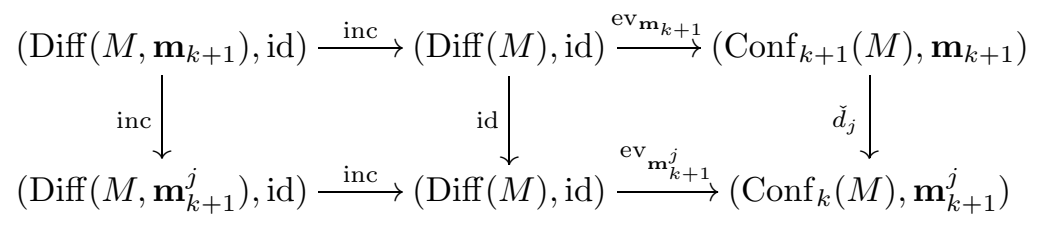

where $\mathbf{e v}_{\mathbf{m}_{k+1}}$ is given by evaluating a diffeomorphism on the $(k+1)$-tuple $\mathbf{m}_{k+1}=$ $\left(m_{0}, \ldots, m_{k}\right)$ and similarly for $\mathrm{ev}_{\mathbf{m}_{k+1}^{j}}$. Using $\partial^{P}$ to denote the connecting homomorphism, the associated diagram of exact homotopy sequences includes

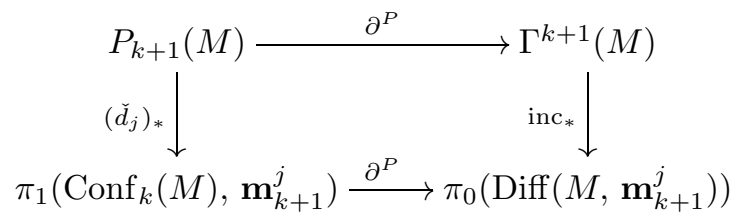

Again, we can describe the connecting homomorphism explicitly. For a path $\lambda=\left(\lambda^{0}, \ldots, \lambda^{k}\right)$ in the configuration space, $\partial^{P}([\lambda])=\left[\Psi_{\lambda}(1)\right]$, where $\Psi_{\lambda}$ is the diffeotopy of $M$ given by simultaneously pushing a small disc containing $m_{i}$ around the path $\lambda^{i}$ for $i=0, \ldots, k$.

Recall that $\delta_{k, j}$ is a path in $\operatorname{Conf}_{k}(M)$ from $\mathbf{m}_{k}$ to $\mathbf{m}_{k+1}^{j}$ and $\theta_{j}: M \rightarrow M$ is a diffeomorphism carrying $\mathbf{m}_{k}$ to $\mathbf{m}_{k+1}^{j}$ whenever $j \leq k$. By Lemma A.4 the following square commutes:

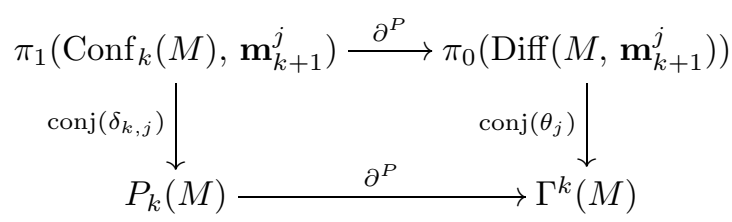

Combining these two squares (and transposing) gives commutativity of the vertical squares in the right-hand cube in (3.2). Commutativity of the remaining vertical squares can be seen directly from the descriptions of the homomorphisms involved.

The groups $\pi_{1}\left(M-\mathbf{m}_{k}, m_{k}\right), k \geq 1$, form a $\Delta$-group, which we denote $\underline{\pi}(M)$, with the maps $d_{j}^{\pi}$ as the face maps. In the case of the sphere, $\underline{\pi}\left(S^{2}\right) \cong \bar{F}\left[S^{1}\right]$, Milnor's free group construction on the simplicial 1-sphere, see for example [1, Section 6. 
Given any $\Delta$-group $\underline{G}$, we may consider the Moore loop space $\Omega \underline{G}$ given by

$$
\Omega \underline{G}_{k}=\left\{g \in G_{k+1}: d_{k+1}(g)=1 \text { and } d_{0} \circ \cdots \circ d_{k}(g)=1\right\},
$$

with face maps $d_{j}^{\Omega G}=d_{j+1}^{G}$. In the case of $\underline{P}\left(S^{2}\right)$ and $\underline{\Gamma}\left(S^{2}\right)$, the zeroth groups are trivial so the $k$ th group of the loop space coincides with the kernel of $d_{k}$. Thus our commutative diagram (3.2) gives us epimorphisms of $\Delta$-groups:

$$
\underline{\pi}\left(S^{2}\right) \rightarrow \Omega \underline{P}\left(S^{2}\right) \text { and } \underline{\pi}\left(S^{2}\right) \rightarrow \Omega \underline{\Gamma}\left(S^{2}\right) .
$$

In Section 3.2 of [3] we show that these morphisms are also injective in degrees $\geq 4$ (and hence we show, in Section 4.1 of that paper, that they induce isomorphisms of homotopy groups).

\section{IsOmorphism BETWEen the BRAID AND MAPPING Class GROUP CASE}

In this section, and in our second paper, we concentrate on the pure braid group $P_{k}(M)$ and the pure mapping class group $\Gamma^{k}(M)$. This is because we wish to consider intersections of kernels of face maps, and we know from Proposition 2.6 and Proposition 2.11 that braids and mapping classes that lie in the kernels of three distinct face maps are pure.

In what follows, $T$ denotes the torus and $K$ the Klein bottle.

4.1. The Moore chain complex of a $\Delta$-group. Let $\underline{G}=\left\{G_{k}\right\}_{k \geq 0}$ be a $\Delta$-group. As before, we define

$$
\mathrm{K}_{i}\left(G_{k}\right):=\operatorname{Ker}\left[d_{i}: G_{k} \rightarrow G_{k-1}\right] .
$$

The Moore chain complex $\mathrm{N}(\underline{G})$ of $\underline{G}$ has the $k$ th group

$$
\mathrm{N}\left(G_{k}\right):=\bigcap_{i=1}^{k} \operatorname{Ker}\left[d_{i}: G_{k} \rightarrow G_{k-1}\right]=\bigcap_{k=1}^{k} \mathrm{~K}_{i}\left(G_{k}\right)
$$

and a differential given by $d_{0}$. The face map identity guarantees that $d_{0}\left(\mathrm{~N}\left(G_{k}\right)\right) \subseteq$ $\mathrm{N}\left(G_{k-1}\right)$ and that the square of the differential is trivial.

We use $\mathrm{Z}\left(G_{k}\right)$ to denote the group of $k$-dimensional boundaries in the Moore chain complex. Thus

$$
\mathrm{Z}\left(G_{k}\right)=\bigcap_{i=0}^{k} \mathrm{~K}_{i}\left(G_{k}\right) .
$$

In the case $\underline{G}=\underline{P}(M)$, the $k$-dimensional cycles are the braids on $k+1$ strings that become trivial when any one of the strings is removed. This is the well-known Brunnian braid group, $\operatorname{Brun}\left(P_{k+1}(M)\right)$. In the case of $\underline{\Gamma}(M)$, the $k$-dimensional cycles are represented by diffeomorphisms fixing $k+1$ points that are isotopic to the identity if any one of the marked points is forgotten. This is the Brunnian mapping class group which we denote $\operatorname{Brun}\left(\Gamma^{k+1}(M)\right.$ ). (The Brunnian braid group is usually defined to be the subgroup of the full braid group $B_{k+1}(M)$ consisting of all braids that become trivial when any one of the strands is removed; by Proposition 2.6. this coincides with $\operatorname{Brun}\left(P_{k+1}(M)\right)$ for all $k \geq 2$. Likewise for the mapping class group, by Proposition 2.11)

The homology sets $\mathrm{Z}\left(G_{k}\right) / d_{0}\left(\mathrm{~N}\left(G_{k+1}\right)\right)$ of $\mathrm{N}(\underline{G})$ are, by definition, the homotopy sets $\pi_{k}(\underline{G})$ of $\underline{G}$. Note that in general, $d_{0}\left(\mathrm{~N}\left(G_{k+1}\right)\right)$ is not a normal subgroup of $\mathrm{Z}\left(G_{k}\right)$, so these sets do not necessarily have a group structure. 
Lemma 4.1. For any surface $M$ and $k \geq 0$, the subgroups $d_{0}\left(\mathrm{~N}\left(P_{k+2}(M)\right)\right) \leq$ $\mathrm{Z}\left(P_{k+1}(M)\right)$ and $d_{0}\left(\mathrm{~N}\left(\Gamma^{k+2}(M)\right)\right) \leq \mathrm{Z}\left(\Gamma^{k+1}(M)\right)$ are normal, and so $\pi_{k}(\underline{P}(M))$ and $\pi_{k}(\underline{\Gamma}(M))$ are groups.

Proof. For any $\Delta$-group $\underline{G}$, the group $d_{0}\left(\mathrm{~N}\left(G_{k+1}\right)\right)$ is normal in $\operatorname{Im}\left[d_{0}: G_{k+1} \rightarrow\right.$ $\left.G_{k}\right]$; see for example Lemma 4.1.2 in [1. Thus it suffices to show that

$$
\begin{aligned}
& \mathrm{Z}\left(P_{k+1}(M)\right) \subseteq \operatorname{Im}\left[d_{0}: P_{k+2}(M) \rightarrow P_{k+1}(M)\right], \\
& \mathrm{Z}\left(\Gamma^{k+1}(M)\right) \subseteq \operatorname{Im}\left[d_{0}: \Gamma^{k+2}(M) \rightarrow \Gamma^{k+1}(M)\right] .
\end{aligned}
$$

The first assertion follows from the fact that $d_{0}: P_{k+2}(M) \rightarrow P_{k+1}(M)$ is surjective, as seen from the top line of (3.5). The second assertion follows similarly from (3.6).

4.2. The homotopy type of the diffeomorphism groups. Here, we recall Earle-Eells's and Earle-Schatz's results about the contractibility of the diffeomorphism groups of surfaces.

Below, $\operatorname{Diff}_{0}(M)$ denotes the subgroup of Diff $(M)$ consisting of all diffeomorphisms that are isotopic to the identity. Alternatively, this can be described as the path-component of the identity in $\operatorname{Diff}(M)$.

Theorem 4.2 (Corollary $1 \mathrm{E}$ in [8]). Let $M$ be a closed surface. Then $\operatorname{Diff}_{0}(M)$ is contractible unless $M$ is $S^{2}, T, \mathbb{R} P^{2}$ or $K$. In the exceptional cases we have

$$
\begin{aligned}
\operatorname{Diff}_{0}\left(S^{2}\right) & \simeq S O(3), \\
\operatorname{Diff}_{0}(T) & \simeq T \\
\operatorname{Diff}_{0}\left(\mathbb{R} P^{2}\right) & \simeq S O(3), \\
\operatorname{Diff}_{0}(K) & \simeq S O(2) .
\end{aligned}
$$

Now suppose that $M$ is a surface with boundary. Let $\operatorname{Diff}^{\mathrm{ES}}(M)$ denote the group of diffeomorphisms of $M$ that map each boundary component to itself, preserving orientation. Note that if $M$ is orientable, then these diffeomorphisms are automatically orientation-preserving. Again, Diff ${ }_{0}^{\mathrm{ES}}(M)$ denotes the group of diffeomorphisms that are isotopic to the identity.

Theorem 4.3 (Theorem $1 \mathrm{C}$ in 9 ). Let $M$ be a surface with boundary. Then $\operatorname{Diff}_{0}^{\mathrm{ES}}(M)$ is contractible unless $M$ is the disc, annulus or Möbius strip. In each of these exceptional cases, $\operatorname{Diff}_{0}^{\mathrm{ES}}(M) \simeq S O(2)$.

Theorem 4.4 (Theorem 1D in [9]). If $M$ is a surface with boundary, then $\operatorname{Diff}_{0}(M)$ is contractible.

Corollary 4.5. Let $M$ be a surface with $k \geq 1$ marked points. Then $\operatorname{Diff}_{0}\left(M, \mathbf{m}_{k}\right)$ is contractible unless $M=S^{2}$ and $k \in\{1,2\}$ or $M=\mathbb{R} P^{2}$ and $k=1$. In these exceptional cases, $\operatorname{Diff}_{0}\left(M, \mathbf{m}_{k}\right) \simeq S O(2)$.

Proof. Suppose first that $M$ is a closed surface. Let $\hat{M}$ be the surface obtained from $M$ by removing a small disc around each marked point. If $M$ is orientable, then $\operatorname{Diff}\left(M, \mathbf{m}_{k}\right)$ is homotopy equivalent to $\operatorname{Diff}^{\mathrm{ES}}(\hat{M})$, so in particular $\operatorname{Diff}_{0}\left(M, \mathbf{m}_{k}\right)$ is homotopy equivalent to $\operatorname{Diff}_{0}^{\mathrm{ES}}(\hat{M})$. If $M$ is not orientable, then $\operatorname{Diff}\left(M, \mathbf{m}_{k}\right)$ is homotopy equivalent to $\operatorname{Diff}^{ \pm \mathrm{ES}}(\hat{M})$, the group of diffeomorphisms of $\hat{M}$ that map 
each boundary component to itself, but that need not preserve the orientation of the boundary components. There is a fibration

$$
\operatorname{Difff}^{\mathrm{ES}}(\hat{M}) \longrightarrow \operatorname{Diff}^{ \pm \mathrm{ES}}(\hat{M}) \longrightarrow(\mathbb{Z} / 2)^{k}
$$

given by recording the orientation change at each boundary component. It follows that $\operatorname{Diff}_{0}^{ \pm \mathrm{ES}}(\hat{M})$ has the same homotopy type as $\operatorname{Diff}_{0}^{\mathrm{ES}}(\hat{M})$. We can conclude that $\operatorname{Diff}_{0}\left(M, \mathbf{m}_{k}\right)$ is homotopy equivalent to $\operatorname{Diff}_{0}^{\mathrm{ES}}(\hat{M})$, as in the orientable case. The result now follows from Theorem 4.3 since the cases $M=S^{2}, k=1, M=S^{2}, k=2$ and $M=\mathbb{R} P^{2}, k=1$ correspond respectively to the cases $\hat{M}=\operatorname{disc}, \hat{M}=$ annulus and $\hat{M}=$ Möbius strip.

Suppose now that $M$ is a surface with boundary. Since $M$ has boundary, $M$ and $M-\mathbf{m}_{k-1}$ are $K(\pi, 1)$ spaces for all $k \geq 2$. It follows inductively from the Fadell-Neuwirth fibration [10]

$$
M-\mathbf{m}_{k-1} \longrightarrow \operatorname{Conf}_{k}(M) \longrightarrow \operatorname{Conf}_{k-1}(M)
$$

that $\operatorname{Conf}_{k}(M)$ is a $K(\pi, 1)$ for all $k \geq 1$. From the evaluation fibration

$$
\operatorname{Diff}\left(M, \mathbf{m}_{k}\right) \longrightarrow \operatorname{Diff}(M) \stackrel{\mathrm{ev}_{\mathbf{m}_{k}}}{\longrightarrow} \operatorname{Conf}_{k}(M),
$$

it now follows via Theorem 4.4 that $\operatorname{Diff}_{0}\left(M, \mathbf{m}_{k}\right)$ is contractible.

4.3. Isomorphism between the braid and the mapping class group setting. Before we begin, we record for later use an algebraic lemma that is proved by a straightforward diagram chase.

Lemma 4.6. Suppose that we have the following commutative diagram of groups and group homomorphisms with exact rows:

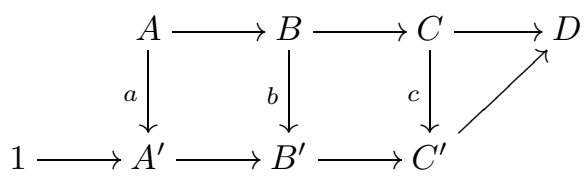

If the map $a$ is an isomorphism, then the induced map $\operatorname{Ker}(b) \rightarrow \operatorname{Ker}(c)$ is also an isomorphism.

Theorem 4.7. Let $M$ be any surface.

(a) There is an isomorphism of Moore chain complexes $\mathrm{N}(\underline{P}(M)) \cong \mathrm{N}(\underline{\Gamma}(M))$ in all degrees

$$
k \geq\left\{\begin{array}{lll}
4 & \text { if } \quad M=S^{2} \\
3 & \text { if } \quad M=\mathbb{R} P^{2}, \\
1 & & \text { for all other } M .
\end{array}\right.
$$

(b) There are isomorphisms $\mathrm{Z}\left(P_{k+1}(M)\right) \cong \mathrm{Z}\left(\Gamma^{k+1}(M)\right)$ and $\pi_{k}(\underline{P}(M)) \cong$ $\pi_{k}(\underline{\Gamma}(M))$ in degrees

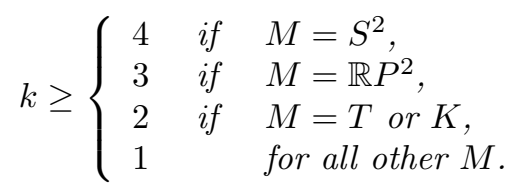


Proof. (a) Consider the diagram of fibrations (3.7). By Corollary 4.5, for $k \geq 1$ in the general case, $k \geq 3$ if $M=S^{2}$ and $k \geq 2$ if $M=\mathbb{R} P^{2}$, the groups $\pi_{1}\left(\operatorname{Diff}\left(M, \mathbf{m}_{k+1}\right)\right)$ and $\pi_{1}\left(\operatorname{Diff}\left(M, \mathbf{m}_{k+1}^{i}\right)\right)$ are trivial. Thus, the associated diagram of exact sequences (again with $\mathrm{C}_{k}$ for $\operatorname{Conf}_{k}(M)$ ) ends with

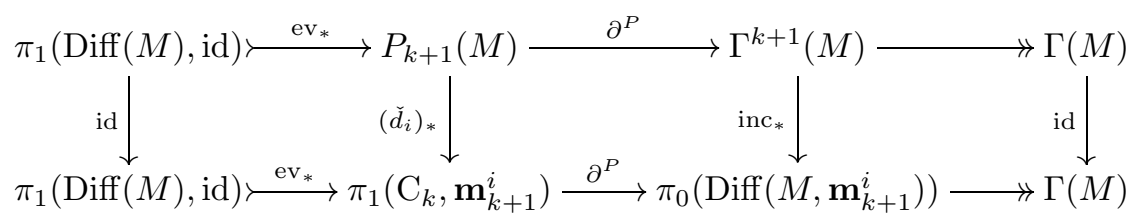

As in Section 3. by conjugating by $\delta_{k, i}$ and $\theta_{i}$ respectively, the diagram of long exact sequences becomes

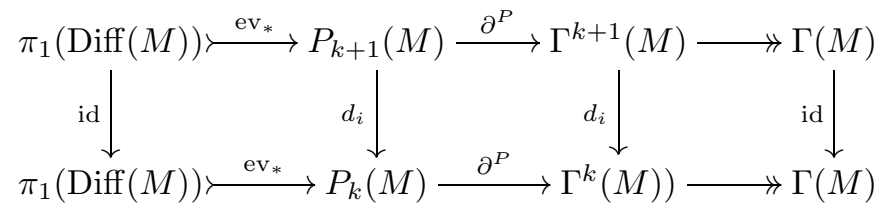

Setting $i=k$ and applying Lemma 4.6 yields an isomorphism

$$
\mathrm{K}_{k}\left(P_{k+1}(M)\right) \longrightarrow \mathrm{K}_{k}\left(\Gamma^{k+1}(M)\right)
$$

Thus, provided that $k \geq 2$ in the general case, $k \geq 4$ if $M=S^{2}$ and $k \geq 3$ if $M=\mathbb{R} P^{2}$, we have a commutative diagram:

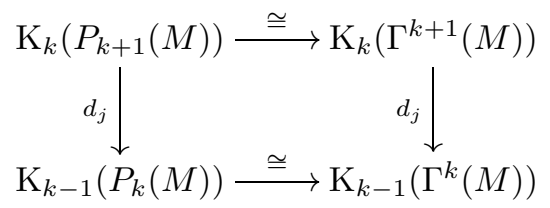

Since

$$
\begin{aligned}
\mathrm{N}\left(P_{k+1}(M)\right) & =\bigcap_{j=1}^{k} \mathrm{~K}_{j}\left(P_{k+1}(M)\right) \\
& =\bigcap_{j=1}^{k-1} \operatorname{Ker}\left[\left.d_{j}\right|_{\mathrm{K}_{k}\left(P_{k+1}(M)\right)}\right],
\end{aligned}
$$

and an analogous statement holds for the mapping class groups, diagram (4.3) gives the isomorphism of Moore chain complexes for these $k$. The isomorphism $\mathrm{N}\left(P_{2}(M)\right) \cong \mathrm{N}\left(\Gamma^{2}(M)\right)$ for the general case is given by (4.2), with $k$ set equal to 1 .

(b) General case: The isomorphisms in degrees $\geq 2$ follow from part (a). The fact that $\mathrm{Z}\left(P_{2}(M)\right) \cong \mathrm{Z}\left(\Gamma^{2}(M)\right)$ follows from the commutative diagram (a subdiagram of (4.1)):

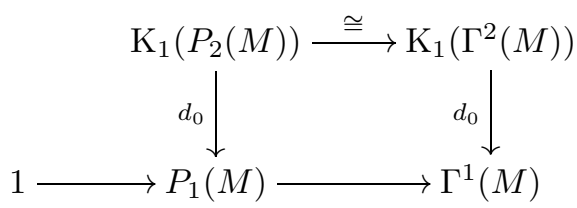

From (a) we know that $d_{0}\left(\mathrm{~N}\left(P_{3}(M)\right)\right) \cong d_{0}\left(\mathrm{~N}\left(\Gamma^{3}(M)\right)\right)$, so we deduce that $\pi_{1}(\underline{P}(M))$ is isomorphic to $\pi_{1}(\underline{\Gamma}(M))$. 
Case of $S^{2}$. The isomorphisms in degrees $\geq 5$ follow from (a). The fact that $\mathrm{Z}\left(P_{5}\left(S^{2}\right)\right) \cong \mathrm{Z}\left(\Gamma^{5}\left(S^{2}\right)\right)$ follows from diagram (4.3) in exactly the same way as we obtained $\mathrm{N}\left(P_{5}\left(S^{2}\right)\right) \cong \mathrm{N}\left(\Gamma^{5}\left(S^{2}\right)\right)$. The fact that $d_{0}\left(\mathrm{~N}\left(P_{6}\left(S^{2}\right)\right)\right) \cong d_{0}\left(\mathrm{~N}\left(\Gamma^{6}\left(S^{2}\right)\right)\right)$ follows from (a); so, we can deduce that $\pi_{4}\left(\underline{P}\left(S^{2}\right)\right) \cong \pi_{4}\left(\underline{\Gamma}\left(S^{2}\right)\right)$.

Case of $\mathbb{R} P^{2}$. The arguments are the same as for the sphere case.

Case of $T$ or $K$. The isomorphisms follow from (a).

Because $\mathrm{Z}\left(P_{k+1}(M)\right)=\operatorname{Brun}\left(P_{k+1}(M)\right)$ and $\mathrm{Z}\left(\Gamma^{k+1}(M)\right)=\operatorname{Brun}\left(\Gamma^{k+1}(M)\right)$, combining part (b) of the theorem with Theorem 1.1 in [1] gives the following corollary.

Corollary 4.8. For all $k \geq 4$ we have $\pi_{k}\left(\underline{\Gamma}\left(S^{2}\right)\right) \cong \pi_{k}\left(S^{2}\right)$.

\section{Appendix A. Deferred proofs}

A.1. The evaluation map is a fibration. The proposition below is asserted in the literature 10, but as we were unable to meet a referee's challenge to cite a proof we provide one here, relating to the proofs of [4, Theorem 3.2], [13, Lemma 1.35] for homeomorphism groups. We are pleased to acknowledge the assistance of Fred Cohen in tracking down the elusive sources. Let us begin by recalling what Steenrod calls the bundle structure theorem.

Lemma A.1 ([17, [19, p.31]). Let $G$ be a topological group acting transitively on a space $X$, and let $\mathrm{ev}: G \rightarrow X$ denote evaluation at the basepoint $x_{0}$ of $X$. If $x_{0}$ has a neighbourhood on which ev admits a section, then ev is a fiber bundle projection.

To apply this, let $D_{\rho}$ be the open disc of radius $\rho$ centred at the origin, with $D=D_{1}$, and let $\operatorname{Diff}_{\mathrm{c}}(D)$ be its topological group of compactly-supported diffeomorphisms (with the compact-open topology), having $\operatorname{Diff}_{\mathrm{c}, \text { id }}(D)$ as its pathcomponent of the identity.

Lemma A.2. The evaluation-at-the-origin map ev : Diff , id $_{\text {id }}(D) \rightarrow$ D has a smooth section in a neighbourhood of the origin.

Proof. As in the proof of [16. Lemma 5.2] (where for convenience we take $r=$ $1 / 2 \sqrt{2})$, we begin with a smooth function $\sigma: \mathbb{R}_{\geq 0} \rightarrow[0,1]$ such that $\sigma([0,1 / 8])=1$, $\sigma\left(\left[1 / 2, \infty[)=0\right.\right.$ and for all $u \geq 0$ we have $\left|\sigma^{\prime}(u)\right|<8$. Then [16, Lemma 5.4] shows the existence of arbitrarily small $\varepsilon>0$ such that, for any $a \in D_{\varepsilon}$, the map

$$
F_{a}: x \longmapsto x+\sigma\left(\|x\|^{2}\right) a
$$

is a diffeomorphism of $\mathbb{R}^{2}$ (isotopic to the identity). Evidently, $F_{a}$ varies smoothly with $a$ and $F_{a}(0)=a$. Because its support lies in $\bar{D}_{1 / \sqrt{2}}$, the diffeomorphism $F_{a}$ restricts to a compactly-supported diffeomorphism of $D$.

Hence, $a \mapsto F_{a}$ serves as a smooth section of ev on $D_{\varepsilon}$.

These lemmas combine to give the following result.

Proposition A.3. For any surface $M$ and any $k \geq 1$, the evaluation map

$$
\mathrm{ev}_{\mathbf{m}_{k}}: \operatorname{Diff}(M) \rightarrow \operatorname{Conf}_{k}(M)
$$

is a locally trivial fibration. 
Proof. By Lemma A.1, it suffices to exhibit a section for $\mathrm{ev}_{\mathbf{m}_{k}}$ in a neighbourhood of $\mathbf{m}_{k}$. For $i=0, \ldots, k-1$, let $V_{i}$ be a disc neighbourhood of $m_{i}$ centred at $m_{i}$ and $W_{i}$ its corresponding $\varepsilon$-neighbourhood on which there is a section $s_{i}: W_{i} \rightarrow$ $\operatorname{Diff}_{\mathrm{c}, \text { id }}\left(V_{i}\right)$ as in Lemma A.2 We may assume that the $V_{i}$ are mutually disjoint; consequently, $W=\prod_{i=0}^{k-1} W_{i}$ is an open neighbourhood of $\mathbf{m}_{k}$ in $\operatorname{Conf}_{k}(M)$.

Observe that any diffeomorphism in $\operatorname{Diff}_{\mathrm{c}}\left(V_{i}\right)$ extends by the identity map outside $V_{i}$, giving an inclusion of $\operatorname{Diff}_{\mathrm{c}}\left(V_{i}\right)$ in $\operatorname{Diff}(M)$. Let $y_{i} \in W_{i}$. Now, as $i$ varies, the supports of the diffeomorphisms $s_{i}\left(y_{i}\right)$ are disjoint because the $V_{i}$ are disjoint. Thus, for any $\mathbf{y}=\left(y_{0}, \ldots, y_{k-1}\right) \in W$, the $k$ diffeomorphisms $s_{i}\left(y_{i}\right)$ commute. Their product is then the image of $\mathbf{y}$ under the desired section $s: W \rightarrow \operatorname{Diff}(M)$ of $\mathrm{ev}_{\mathbf{m}_{k}}$ on $W$.

A.2. The paths $\delta_{k, i}$ and diffeomorphisms $\theta_{i}$. For completeness, we record an explicit description of the paths $\delta_{k, i}$ and the diffeomorphism $\theta_{i}$ used to define the face maps for braid groups and mapping class groups. The whisker, a smooth embedding $\rho: \mathbb{R} \rightarrow M-\partial M$ with the property that the closure of $\operatorname{Im} \rho$ is homeomorphic to an interval, determines the marked points in $M$ by means of $m_{i}=\rho(i)$ whenever $i \in \mathbb{N}_{+}=\{0,1, \ldots\}$. Also recall that $\mathbf{m}_{k}=\left(m_{0}, \ldots, m_{k-1}\right) \in \operatorname{Conf}_{k}(M)$ and $\mathbf{m}_{k}^{i}=\left(m_{0}, \ldots, \hat{m}_{i}, \ldots, m_{k-1}\right) \in \operatorname{Conf}_{k-1}(M)$.

First, we have that $\delta_{k, i}: I \rightarrow \operatorname{Conf}_{k}(M)$ is given by

$$
\delta_{k, i}(t)=\left(\delta_{i}\left(m_{0}, t\right), \ldots, \delta_{i}\left(m_{k-1}, t\right)\right)
$$

where

$$
\begin{gathered}
\delta_{i}: \rho\left(\mathbb{N}_{+}\right) \times I \longrightarrow M, \\
\delta_{i}(\rho(j), t)=\left\{\begin{array}{cc}
\rho(j) & \text { if } j<i, \\
\rho(j+t) & \text { if } j \geq i .
\end{array}\right.
\end{gathered}
$$

Evidently, whenever $i \leq k, \delta_{k, i}$ is a path from $\mathbf{m}_{k}$ to $\mathbf{m}_{k+1}^{i}$. If we rewrite this in the form $\delta_{k, i}=\left(\delta_{i}^{0}, \ldots, \delta_{i}^{k-1}\right)$, where $\delta_{i}^{j}(t)=\delta_{i}(\rho(j), t)$, then clearly $\delta_{k, i}=\check{d}_{k} \circ \delta_{k+1, i}$, where $\breve{d}_{k}$ denotes the map of configuration spaces given by forgetting the $k$ th point. Moreover, $\delta_{k, k}$ is the constant path on $\mathbf{m}_{k}=\mathbf{m}_{k+1}^{k}$.

Now we move on to the diffeomorphism $\theta_{i}$. Recall that for $i \leq k$ we want the diffeomorphism $\theta_{i}$ to carry $\mathbf{m}_{k}$ onto $\mathbf{m}_{k+1}^{i}$, preserving the order of the points. Our choice of $\theta_{i}$ will be diffeotopic to the identity if the marked points are forgotten. In order to relate it to the path $\delta_{k, i}: I \rightarrow \operatorname{Conf}_{k}(M)$ from $\mathbf{m}_{k}$ to $\mathbf{m}_{k+1}^{i}$ that plays a similar role in the braid groups, we specify a diffeotopy $\Theta_{i}$ starting at $\operatorname{id}_{\operatorname{Im} \rho}$ and extending $\delta_{i}$, namely

defined by

$$
\Theta_{i}: \operatorname{Im} \rho \times I \longrightarrow \operatorname{Im} \rho
$$

$$
\Theta_{i}(\rho(x), t)=\left\{\begin{array}{cl}
\rho(x) & \text { if } x<i-1, \\
\rho(x+t(x-(i-1))) & \text { if } i-1 \leq x<i, \\
\rho(x+t) & \text { if } x \geq i .
\end{array}\right.
$$

Thus, whenever $i \leq k, \Theta_{i}$ slides the points $\mathbf{m}_{k}$ along $\operatorname{Im} \rho$ to $\mathbf{m}_{k+1}^{i}$. Evidently, $\Theta_{i}$ does begin at id, and ends at $\theta_{i}: \operatorname{Im} \rho \rightarrow \operatorname{Im} \rho$ defined by

$$
\theta_{i}(\rho(x))=\left\{\begin{array}{cl}
\rho(x) & \text { if } x<i-1, \\
\rho(2 x-(i-1)) & \text { if } i-1 \leq x<i, \\
\rho(x+1) & \text { if } x \geq i .
\end{array}\right.
$$

Whenever $i \leq k, \theta_{i}\left(\mathbf{m}_{k}\right)=\mathbf{m}_{k+1}^{i}$ as we intended. 
This diffeotopy extends in a natural way to a diffeotopy

$$
\Theta_{i}: M \times I \longrightarrow M
$$

that we may assume is the identity (at all times) outside a tubular neighbourhood of $\operatorname{Im} \rho$. Note also that, for $j \in\{0,1, \ldots, k-1\}$,

$$
\Theta_{i}\left(m_{j}, t\right)=\delta_{i}\left(m_{j}, t\right) .
$$

For reference, we record these facts as follows.

Lemma A.4. (a) $\Theta_{i}: M \times I \rightarrow M$ is a diffeotopy from $\operatorname{id}_{M}$ to $\theta_{i}: M \rightarrow M$.

(b) $\left.\Theta_{i}\right|_{\left(\rho\left(\mathbb{N}_{+}\right) \times I\right)}=\delta_{i}$.

(c) For $i \leq k,\left.\Theta_{i}\right|_{\left(\mathbf{m}_{k} \times I\right)}=\delta_{k, i}$.

(d) For $i \leq k, \delta_{k, i}=\check{d}_{k} \circ \delta_{k+1, i}$.

A.3. Proof of compatibility in the full case. Here we provide a rigorous proof that the $\Delta$-structure for the full braid groups is compatible with that for the full mapping class groups. It is convenient to think of the braid groups as $\operatorname{Br}_{k}(M)=\pi_{1}\left(\Sigma_{k} \backslash \operatorname{Conf}_{k}(M)\right)$. Recall from Section 2 that there are isomorphisms $g: \operatorname{Br}_{k}(M) \rightarrow B_{k}(M)$ and the $\Delta$-structure we defined for $\left\{B_{k+1}(M)\right\}_{k \geq 0}$ induces a $\Delta$-structure for $\left\{\operatorname{Br}_{k+1}(M)\right\}_{k \geq 0}$ via these isomorphisms.

Recall from the start of Section 3 that there is a long exact sequence

$$
\cdots \longrightarrow \pi_{1}(\operatorname{Diff}(M)) \longrightarrow \operatorname{Br}_{k}(M) \stackrel{\partial}{\longrightarrow} \Gamma^{(k)}(M) \longrightarrow \Gamma(M) \rightarrow 1 .
$$

Theorem A.5. The connecting homomorphism in this exact sequence induces a map $\underline{\operatorname{Br}}(M) \rightarrow \underline{\Gamma}^{\mathrm{sym}}(M)$ of crossed $\Delta$-groups.

Proof. We need to check that the map $\partial: \operatorname{Br}_{k+1}(M) \rightarrow \Gamma^{(k+1)}(M)$ commutes with the face maps and is compatible with the maps to the symmetric groups. We begin with a preliminary lemma.

Lemma A.6. (a) For any $\phi \in \operatorname{Diff}(M)$, we have $\mathrm{ev}_{\mathbf{m}_{k}}\left(\theta_{i} \odot \phi\right)=\mathrm{ev}_{\mathbf{m}_{k+1}^{i}}(\phi)$.

(b) For every $\phi \in \operatorname{Diff}(M)$ and $\psi \in \operatorname{Diff}\left(M,\left[\mathbf{m}_{k}\right]\right)$ we have

$$
\mathrm{ev}_{\mathbf{m}_{k}}(\psi \odot \phi)=\mu(\psi) \cdot \mathrm{ev}_{\mathbf{m}_{k}}(\phi) .
$$

The product on the right-hand side denotes the natural symmetric group action on $\operatorname{Conf}_{k}(M)$, as defined by equation (2.2).

Proof. (a) This is clear since $\theta_{i}$ sends $\mathbf{m}_{k}$ to $\mathbf{m}_{k+1}^{i}$, preserving the order of the points.

(b) Let $n_{i}=m_{i} \cdot \phi \in M$. Then

$$
\begin{aligned}
\mathrm{ev}_{\mathbf{m}_{k}}(\psi \odot \phi) & =\left(\left(m_{0}, \ldots, m_{k-1}\right) \cdot \psi\right) \cdot \phi \\
& =\left(m_{0 \cdot \mu(\psi)}, \ldots, m_{(k-1) \cdot \mu(\psi)}\right) \cdot \phi \\
& =\left(m_{0 \cdot \mu(\psi)} \cdot \phi, \ldots, m_{(k-1) \cdot \mu(\psi)} \cdot \phi\right) \\
& =\left(n_{0 \cdot \mu(\psi)}, \ldots, n_{(k-1) \cdot \mu(\psi)}\right)
\end{aligned}
$$

and

$$
\begin{aligned}
\mu(\psi) \cdot \mathrm{ev}_{\mathbf{m}_{k}}(\phi) & =\mu(\psi) \cdot\left(m_{0} \cdot \phi, \ldots, m_{k-1} \cdot \phi\right) \\
& =\mu(\psi) \cdot\left(n_{0}, \ldots, n_{k-1}\right) \\
& =\left(n_{0 \cdot \mu(\psi)}, \ldots, n_{(k-1) \cdot \mu(\psi)}\right) .
\end{aligned}
$$


Let $\gamma:(I,\{0,1\}) \rightarrow\left(\Sigma_{k+1} \backslash \operatorname{Conf}_{k+1}(M),\left[\mathbf{m}_{k+1}\right]\right)$ represent an element $[\gamma] \in$ $\operatorname{Br}_{k+1}(M)$. We wish to show that

(I) $\mu(\partial[\gamma])=(\pi \circ g)[\gamma]$ and

(II) $d_{i} \partial[\gamma]=\partial d_{i}[\gamma]$.

Let

$$
\begin{aligned}
& \tilde{\gamma}: \quad(I, 0) \longrightarrow\left(\operatorname{Conf}_{k+1}(M), \mathbf{m}_{k+1}\right), \\
& \bar{\gamma}:(I, 0) \longrightarrow(\operatorname{Diff}(M), \operatorname{id})
\end{aligned}
$$

be lifts of $\gamma$; in particular, by unique path-lifting for covering spaces, $\bar{\gamma}$ is also a lift of $\widetilde{\gamma}$.

Lemma A.7. $\mu(\bar{\gamma}(1))=\pi(\widetilde{\gamma})$.

Proof. Recall that $\pi(\widetilde{\gamma})$ is defined by

$$
\widetilde{\gamma}(1)=\left(m_{0 \cdot \pi(\widetilde{\gamma})}, \ldots, m_{k \cdot \pi(\widetilde{\gamma})}\right),
$$

but

$$
\begin{aligned}
\widetilde{\gamma}(1) & =\left(\operatorname{ev}_{\mathbf{m}_{k+1}}(\bar{\gamma})\right)(1) \\
& =\left(m_{0}, \ldots, m_{k}\right) \cdot \bar{\gamma}(1) \\
& =\left(m_{0 \cdot \mu(\bar{\gamma}(1))}, \ldots, m_{k \cdot \mu(\bar{\gamma}(1))}\right) .
\end{aligned}
$$

This suffices to prove (I) since, by the definition of the connecting homomorphism,

$$
\partial[\gamma]=[\bar{\gamma}(1)] \text { and } g[\gamma]=[\widetilde{\gamma}] \text {. }
$$

For the proof of (II), we start by identifying $d_{i} \partial[\gamma]$. From (2.3) and the expression for $\partial[\gamma]$, we have

$$
d_{i} \partial[\gamma]=\left[\theta_{i} \odot \bar{\gamma}(1) \odot \theta_{i \cdot \mu(\bar{\gamma}(1))}^{-1}\right] .
$$

Now let us calculate $\partial d_{i}[\gamma]$. We have that

$$
\partial d_{i}[\gamma]=\left[\overline{d_{i} \gamma}(1)\right]
$$

where $\overline{d_{i} \gamma}:(I, 0) \longrightarrow(\operatorname{Diff}(M)$, id $)$ is a lift of

$$
d_{i} \gamma:(I,\{0,1\}) \longrightarrow\left(\Sigma_{k} \backslash \operatorname{Conf}_{k}(M),\left[\mathbf{m}_{k}\right]\right) .
$$

By the definition of the face maps for $\operatorname{Br}_{k+1}(M), d_{i} \gamma=p \circ\left(d_{i} \widetilde{\gamma}\right)$. Thus, we can calculate $\overline{d_{i} \gamma}$ by lifting $d_{i} \widetilde{\gamma}$ up the fibration $\mathrm{ev}_{\mathbf{m}_{k}}$.

As specified in Section 2.4 there is a diffeotopy

$$
\Theta_{i}: M \times I \longrightarrow M
$$

from the identity to $\theta_{i}$. It will be convenient to think of $\Theta_{i}$ as a path in the diffeomorphism space

$$
\Theta_{i}: I \longrightarrow \operatorname{Diff}(M),
$$

whose inverse path is denoted $\Theta_{i}^{\mathrm{I}}$. For a diffeomorphism $\psi$ of $M$ and a path $\Theta$ : $I \rightarrow \operatorname{Diff}(M)$, we use $\Theta \odot \psi$ to denote the path

$$
(\Theta \odot \psi)(t)=\Theta(t) \odot \psi, \quad t \in I .
$$


Claim. A lift of $d_{i} \tilde{\gamma}$ up the fibration $\mathrm{ev}_{\mathbf{m}_{k}}$ is given by

$$
\Theta_{i} *\left(\theta_{i} \odot \bar{\gamma}\right) *\left(\theta_{i} \odot \bar{\gamma}(1) \odot \theta_{i \cdot \mu(\bar{\gamma}(1))}^{-1} \odot \Theta_{i \cdot \mu(\bar{\gamma}(1))}^{\mathrm{I}}\right),
$$

and hence this element is a valid choice for $\overline{d_{i} \gamma}$.

By the definition of $d_{i}$,

$$
d_{i} \widetilde{\gamma}=\delta_{k, i} * \check{d}_{i} \widetilde{\gamma} *\left(d_{i} \pi(\widetilde{\gamma}) \cdot \delta_{k, i \cdot \pi(\widetilde{\gamma})}^{\mathrm{I}}\right)
$$

To prove the claim, we therefore check:

(i) $\mathrm{ev}_{\mathbf{m}_{k}}\left(\Theta_{i}\right)=\delta_{k, i}$;

(ii) $\operatorname{ev}_{\mathbf{m}_{k}}\left(\theta_{i} \odot \bar{\gamma}\right)=\check{d}_{i} \widetilde{\gamma}$;

(iii) $\mathrm{ev}_{\mathbf{m}_{k}}\left(\theta_{i} \odot \bar{\gamma}(1) \odot \theta_{i \cdot \mu(\bar{\gamma}(1))}^{-1} \odot \Theta_{i \cdot \mu(\bar{\gamma}(1))}^{\mathrm{I}}\right)=d_{i} \pi(\widetilde{\gamma}) \cdot \delta_{k, i \cdot \pi(\widetilde{\gamma})}^{\mathrm{I}} \cdot$

(i) is true by Lemma A.4 (b). To check (ii), observe that

$$
\begin{aligned}
\operatorname{ev}_{\mathbf{m}_{k}}\left(\theta_{i} \odot \bar{\gamma}\right) & =\operatorname{ev}_{\mathbf{m}_{k+1}^{i}}(\bar{\gamma}) \text { by Lemma A.6 (a) } \\
& =\check{d}_{i}(\widetilde{\gamma}) \text { from diagram (3.7). }
\end{aligned}
$$

To obtain (iii), observe that

$$
\begin{aligned}
& \mathrm{ev}_{\mathbf{m}_{k}}\left(\theta_{i} \odot \bar{\gamma}(1) \odot \theta_{i \cdot \mu(\bar{\gamma}(1))}^{-1} \odot \Theta_{i \cdot \mu(\bar{\gamma}(1))}^{\mathrm{I}}\right) \\
& =\mu\left(\theta_{i} \odot \bar{\gamma}(1) \odot \theta_{i \cdot \mu(\bar{\gamma}(1))}^{-1}\right) \cdot \mathrm{ev}_{\mathbf{m}_{k}}\left(\Theta_{i \cdot \mu(\bar{\gamma}(1))}^{\mathrm{I}}\right) \text { by Lemma A.6 (b) } \\
& =\mu\left(\theta_{i} \odot \bar{\gamma}(1) \odot \theta_{i \cdot \mu(\bar{\gamma}(1))}^{-1}\right) \cdot \delta_{k, i \cdot \mu(\bar{\gamma}(1))}^{\mathrm{I}} \text { by Lemma A.4(b) } \\
& =\mu\left(d_{i} \partial[\gamma]\right) \cdot \delta_{k, i \cdot \mu(\bar{\gamma}(1))}^{\mathrm{I}} \text { by (A.2) } \\
& =d_{i} \mu([\bar{\gamma}(1)]) \cdot \delta_{k, i \cdot \mu(\bar{\gamma}(1))}^{\mathrm{I}} \text { by (A.1) and the fact that } \mu \text { commutes } \\
& \text { with } d_{i} \text { (see Theorem 2.9) } \\
& =d_{i} \pi(\widetilde{\gamma}) \cdot \delta_{k, i \cdot \pi(\widetilde{\gamma})}^{\mathrm{I}} \text { by Lemma A.7 }
\end{aligned}
$$

This completes the proof of the claim. Now we have that

$$
\begin{aligned}
\partial d_{i}[\gamma] & =\left[\overline{d_{i} \gamma}(1)\right] \\
& =\left[\Theta_{i} *\left(\theta_{i} \odot \bar{\gamma}\right) *\left(\theta_{i} \odot \bar{\gamma}(1) \odot \theta_{i \cdot \mu(\bar{\gamma}(1))}^{-1} \odot \Theta_{i \cdot \mu(\bar{\gamma}(1))}^{\mathrm{I}}\right)(1)\right] \\
& =\left[\theta_{i} \odot \bar{\gamma}(1) \odot \theta_{i \cdot \mu(\bar{\gamma}(1))}^{-1} \odot \Theta_{i \cdot \mu(\bar{\gamma})}^{\mathrm{I}}(1)\right] \\
& =\left[\theta_{i} \odot \bar{\gamma}(1) \odot \theta_{i \cdot \mu(\bar{\gamma}(1))}^{-1}\right] \\
& =d_{i} \partial[\gamma], \text { as required. }
\end{aligned}
$$

A.4. The face map identity for mapping class groups. We may use the compatibility of the face maps for braid groups and mapping class groups to give a rigorous proof of Proposition 2.8. We prove that the face map identity holds for the groups $\operatorname{Br}_{k}(M)$; thus, it also holds for the groups $B_{k}(M) \cong \operatorname{Br}_{k}(M)$.

When $M$ is a disc, $\operatorname{Diff}(M)$ is contractible (see Theorem 4.4), so the map $\partial$ : $\operatorname{Br}_{k}\left(D^{2}\right) \rightarrow \Gamma^{(k)}\left(D^{2}\right)$ from the long exact sequence (3.1) is an isomorphism. We know that the face map identity holds for $\left\{\operatorname{Br}_{k+1}\left(D^{2}\right)\right\}_{k \geq 0}$ by [1. Since the face maps commute with the isomorphism $\partial$, we can conclude that the face map identity also holds for $\left\{\Gamma^{(k+1)}\left(D^{2}\right)\right\}_{k \geq 0}$.

Now we move on to the general case. We need to check that

$$
d_{j} d_{i}=d_{i} d_{j+1}: \Gamma^{(k+1)}(M) \longrightarrow \Gamma^{(k-1)}(M) \quad \text { for all } i \leq j .
$$


The identity A.3 holds if and only if the following isotopy holds for all $\phi \in$ $\operatorname{Diff}\left(M,\left[\mathbf{m}_{k+1}\right]\right)$ :

$$
\begin{aligned}
& \theta_{j} \odot\left(\theta_{i} \odot \phi \odot \theta_{i \cdot \mu(\phi)}^{-1}\right) \odot \theta_{j \cdot \mu\left(d_{i}(\phi)\right)}^{-1} \\
& \simeq \theta_{i} \odot\left(\theta_{j+1} \odot \phi \odot \theta_{(j+1) \cdot \mu(\phi)}^{-1}\right) \odot \theta_{i \cdot \mu\left(d_{j+1}(\phi)\right)}^{-1}
\end{aligned}
$$

relative to $\mathbf{m}_{k-1}$ (and the boundary).

For (A.4) to hold, it is sufficient that

$$
\theta_{j} \odot \theta_{i} \simeq \theta_{i} \odot \theta_{j+1}
$$

relative to $\mathbf{m}_{k-1}$ and the boundary and

$$
\theta_{j \cdot \mu\left(d_{i}(\phi)\right)} \odot \theta_{i \cdot \mu(\phi)} \simeq \theta_{i \cdot \mu\left(d_{j+1}(\phi)\right)} \odot \theta_{(j+1) \cdot \mu(\phi)}
$$

relative to $\mathbf{m}_{k-1}$ and the boundary.

We know that the face map identity holds for the mapping class groups of a disc, so these two isotopies must hold for the $\theta$ associated to the disc. We can embed the disc with marked points into a general surface $M$ with marked points in such a way that the marked points line up. With this done, the $\theta$ for $M$ are obtained by extending the $\theta$ for the disc by the identity. Thus, we can conclude that the $\theta$ for $M$ also satisfy the isotopies.

\section{REFERENCES}

1. A. J. Berrick, F. R. Cohen, Y. L. Wong and J. Wu: Configurations, braids and homotopy groups, J. Amer. Math. Soc. 19 (2006), 265-326. MR2188127(2007e:20073)

2. A. J. Berrick and E. Hanbury: Simplicial structures and normal forms for mapping class groups and braid groups, preprint (2012).

3. A. J. Berrick, E. Hanbury and J. Wu: Brunnian subgroups of mapping class groups and braid groups, Proc. London Math. Soc. 107 (2013), 875-906.

4. F. R. Cohen: Introduction to configuration spaces and their applications, in Braids: Introductory Lectures on Braids, Configurations and Their Applications, IMS NUS Lect. Notes Series 19, World Scientific (Singapore, 2010), 183-261. MR2605307 (2011f:55034)

5. F. R. Cohen and J. Wu: On braids, free groups and the loop space of the 2-sphere, in Algebraic Topology: Categorical Decompositions, Progress in Math. Tech. 215 (2004), 93105. MR2039761 (2005b:55011)

6. F. R. Cohen and J. Wu: On braid groups and homotopy groups. Groups, homotopy and configuration spaces, Geom. Topol. Monogr., 13 (Coventry, 2008), 169-193. MR2508205 (2010i:55016)

7. A. Connes: Cohomologie cyclique et foncteurs Ext ${ }^{n}$, C. R. Acad. Sci. Paris 296 (1983), 953-958. MR777584 (86d:18007)

8. C. J. Earle and J. Eells: A fibre bundle description of Teichmüller theory, J. Diff. Geom. 3 (1969), 19-43. MR0276999 (43:2737a)

9. C. J. Earle and A. Schatz: Teichmüller theory for surfaces with boundary, J. Diff. Geom. 4 (1970), 169-185. MR0277000 (43:2737b)

10. E. Fadell and L. Neuwirth: Configuration spaces, Math. Scand. 10 (1962) 111-118. MR0141126(25:4537)

11. Z. Fiedorowicz and J.-L. Loday: Crossed simplicial groups and their associated homology, Trans. Amer. Math. Soc. 326 (1991), 57-87. MR998125 (91j:18018)

12. M. W. Hirsch: Differential Topology. Graduate Texts in Math., No. 33. Springer-Verlag (New York-Heidelberg, 1976). MR0448362 (56:6669)

13. C. Kassel and V. Turaev: Braid Groups. With the graphical assistance of Olivier Dodane. Graduate Texts in Math., 247. Springer (New York, 2008). MR2435235(2009e:20082)

14. J. Li and J. Wu: Artin braid groups and homotopy groups, Proc. London Math. Soc. 99 (2009), 521-556. MR2551462(2010k:55020)

15. J.-L. Loday: Homologies diédrale et quaternionique, Adv. in Math. 66 (1987), 119-148. MR.917736 (89e:18024) 
16. R. S. Palais: Natural operations on differential forms, Trans. Amer. Math. Soc. 92 (1959), 125-141. MR0116352 (22:7140)

17. R. S. Palais: Local triviality of the restriction map for embeddings, Comment. Math. Helvetici 34 (1960), 305-312. MR0123338 (23:A666)

18. E. H. Spanier: Algebraic Topology, McGraw-Hill (New York, 1966). MR0210112 (35:1007)

19. N. Steenrod: The Topology of Fibre Bundles, Princeton Univ. Press (Princeton, NJ, 1951). MR0039258(12:522b)

20. J. Wu: A braided simplicial group, Proc. London Math. Soc. 84 (2001), 645-662. MR.1888426 (2003e:20041)

21. J. Wu: Combinatorial descriptions of homotopy groups of certain spaces, Math. Proc. Camb. Phil. Soc. 130 (2001), 489-513. MR1816806 (2003e:55014)

22. W. Zhang: Group operads and homotopy theory, preprint, 2011. arxiv.org/pdf/1111.7090.

Department of Mathematics, National University of Singapore, Singapore

E-mail address: berrick@math.nus.edu.sg

Current address: Yale-NUS College, Singapore 138614, Singapore

E-mail address: berrick@yale-nus.edu.sg

Department of Mathematics, Durham University, Durham DH1 3LE, United Kingdom

E-mail address: elizabeth.hanbury@durham.ac.uk

Department of Mathematics, National University of Singapore, Singapore

E-mail address: matwuj@math.nus.edu.sg 